Communications in Mathematical Physics manuscript No.

(will be inserted by the editor)

\title{
Painlevé transcendent evaluations of finite system density matrices for 1d impenetrable Bosons
}

\author{
P.J. Forrester ${ }^{1}$, N.E. Frankel $^{2}$, T.M. Garoni ${ }^{2}$ and N.S. Witte ${ }^{1,2}$ \\ 1 Department of Mathematics and Statistics, University of Melbourne, Victoria 3010, Aus- \\ tralia. \\ E-mail: P.Forrester@ms.unimelb.edu.au, N.Witte@ms.unimelb.edu.au \\ 2 School of Physics, University of Melbourne, Victoria 3010, Australia. \\ E-mail: n.frankel@physics.unimelb.edu.au, t.garoni@physics.unimelb.edu.au
}

Received: X X 2002 / Accepted: X X 200X

\begin{abstract}
The recent experimental realisation of a one-dimensional Bose gas of ultra cold alkali atoms has renewed attention on the theoretical properties of the impenetrable Bose gas. Of primary concern is the ground state occupation of effective single particle states in the finite system, and thus the tendency for Bose-Einstein condensation. This requires the computation of the density matrix. For the impenetrable Bose gas on a circle we evaluate the density matrix in terms of a particular Painlevé VI transcendent in $\sigma$-form, and furthermore show that the density matrix satisfies a recurrence relation in the number of particles. For the impenetrable Bose gas in a harmonic trap, and with Dirichlet or Neumann boundary conditions, we give a determinant form for the density matrix, a form as an average over the eigenvalues of an ensemble of random matrices, and in special cases an evaluation in terms of a transcendent related to Painlevé V and VI. We discuss how our results can be used to compute the ground state occupations.
\end{abstract}

\section{Introduction}

Recent advances in the experimental physics of Bose-Einstein condensates [14, 15]6] have led to the experimental realisation of a one-dimensional Bose gas of ultra-cold alkali atoms. One expects [39] that the microscopic forces are such that there is an effective one-body confining harmonic potential acting on each atom individually, and an effective infinitely short range contact potential acting between neighbouring atoms. Moreover, in a certain physical regime depending on the ratio of the transverse confinement width to the $s$-wave scattering length, it is argued in [39] that the contact potential can be well approximated by the delta function form $U(|x-y|)=g \delta(|x-y|)$, and furthermore $g \rightarrow \infty$ in the low energy scattering limit. The limit $g \rightarrow \infty$ of the delta function interaction Bose gas is the impenetrable Bose gas, introduced in [12, 32. 
Not surprisingly, there has thus been renewed interest in the theoretical properties of the ground state of the finite system impenetrable Bose gas 39.13. With the 3d Bose gas exhibiting Bose-Einstein condensation, a central question is the tendency of the finite system confined to $1 \mathrm{~d}$ to form a Bose-Einstein condensate. To attack this question is a two step process. First, with the particles confined to the region $\Omega \in \mathbb{R}$ and the ground state wave function $\psi_{0}$ real, it is necessary to compute the one-particle density matrix

$$
\rho_{N}(x ; y)=N \int_{\Omega} d x_{2} \ldots \int_{\Omega} d x_{N} \psi_{0}\left(x, x_{2}, \ldots, x_{N}\right) \psi_{0}\left(y, x_{2}, \ldots, x_{N}\right),
$$

Second, one must solve the eigenvalue problem

$$
\int_{\Omega} \rho_{N}(x ; y) \phi_{k}(y) d y=\lambda_{k} \phi_{k}(x), \quad k \in \mathbb{Z}_{\geq 0} .
$$

Because this integral operator is idempotent, the $\lambda_{j}$ are non-negative, while the trace condition $\int_{\Omega} \rho_{N}(x ; x) d x=N$ implies $\sum_{k} \lambda_{k}=N$. Consequently the $\lambda_{k}$ have the interpretation as occupation numbers of effective single particle states $\phi_{k}(x)$. The simplest case is when $\Omega=[0, L]$ with periodic boundary conditions. The periodicity implies that $\rho_{N}(x ; y)=\rho_{N}(x-y ; 0)$. Thus we have $\phi_{k}(x)=\frac{1}{\sqrt{L}} e^{2 \pi i k x / L}$ and so

$$
\lambda_{k}=\int_{0}^{L} \rho_{N}(x ; 0) e^{2 \pi i k x / L} d x .
$$

However for other geometries and confinements there is no analogue of (1.3) and one must solve (1.2) numerically.

A number of results are available on $\rho_{N}(x ; 0)$ for periodic boundary conditions. In particular Lenard 29] has given $\rho_{N+1}(x ; 0)$ as an $N \times N$ Toeplitz determinant (see (2.5) - below), and subsequently obtained the $N \rightarrow \infty$ asymptotic expansion [31]

$$
\rho_{N}(x ; 0) \sim \rho_{0} A\left(\frac{\pi}{N \sin \left(\pi \rho_{0} x / N\right)}\right)^{1 / 2}, \quad A=\frac{G^{4}(3 / 2)}{\sqrt{2 \pi}}
$$

where $\rho_{0}$ denotes the bulk density and $G(x)$ denotes the Bairn's G-function, valid for $x / N$ fixed. Although the analysis of [31] leading to (1.4) was not rigorous, the setting of the problem as belonging to the asymptotics of Toeplitz determinants with symbols having zeros on $[0,2 \pi)$ was identified, and this work inspired a subsequent rigorous proof [6]. (We remark that the asymptotic form of Toeplitz determinants of this type was first conjectured by Fisher and Hartwig [7, [16. ) The result (1.4) substituted into (1.3) gives $\lambda_{0} \sim c \sqrt{N}$ for a specific $c$ computable from (1.4). Thus for large $N$ the fraction of particles in the zero momentum state is proportional to $\sqrt{N}$. The result (1.4) can also be used to compute the large $N$ behaviour of $\lambda_{k}$ for any fixed $k \geq 0$. For the impenetrable Bose gas confined by a harmonic one-body potential, or indeed in other geometries such as Dirichlet or Neumann boundary conditions, no results of this type are known. All one has is the recent numerical study of Girardeau et al. 13 in the case of the harmonic well, who by a Monte Carlo study of system sizes up to 
$N=10$ obtained the estimate $\lambda_{0} \propto N^{0.59}$ for large $N$. If correct, this result implies the maximum effective single particle state occupation is dependent on the geometry/confining potential.

To further study this issue, we take up the first step in the procedure above to compute the $\lambda_{j}$, and thus provide formulas suitable for the numerical computation of $\rho_{N}(x ; y)$. Four cases are considered - when the domain is a circle (or equivalently periodic boundary conditions); a line with the particles confined by a harmonic one-body potential; and an interval with Dirichlet or Neumann boundary conditions. The Toeplitz determinant formulation in the case of periodic boundary conditions is extended to Hankel determinant forms for $\rho_{N}(x ; y)$ in the other cases (Section 2.2), and a formulation for efficient Monte Carlo evaluations by way of expressing the $\rho_{N}(x ; y)$ as averages over the eigenvalue probability density function (p.d.f.) of certain matrix ensembles is given (Section 2.3). We then give a systematic Fredholm type expansion of $\rho_{N}(x ; y)$ about the density $\rho_{N}(x ; x)$ (Section 2.4).

Beginning in Section 3 we address the issue of closed form evaluations of $\rho_{N}(x ; y)$. In the infinite system there are some celebrated instances of such evaluations. In particular Jimbo et al. 22 related the problem of evaluating $\rho_{\infty}(x ; 0)$ to integrable systems theory, and consequently were able to derive the formula

$$
\rho_{\infty}(x ; 0)=\rho_{0} \exp \left(\int_{0}^{\pi \rho_{0} x} \sigma_{V}(t) \frac{d t}{t}\right),
$$

where $\sigma_{V}$ satisfies the non-linear equation

$$
\left(x \sigma_{V}^{\prime \prime}\right)^{2}+4\left(x \sigma_{V}^{\prime}-\sigma_{V}-1\right)\left(x \sigma_{V}^{\prime}-\sigma_{V}+\left(\sigma_{V}^{\prime}\right)^{2}\right)=0
$$

subject to the $x \rightarrow 0$ boundary condition

$$
\sigma_{V}(x) \underset{x \rightarrow 0}{\sim}-\frac{x^{2}}{3}+\frac{x^{3}}{3 \pi}+\mathrm{O}\left(x^{4}\right) .
$$

The differential equation (1.6) is an example of the so-called Jimbo-Miwa-Okamoto $\sigma$-form of the Painlevé $\mathrm{V}$ equation, the latter being essentially the differential equation obeyed by the Hamiltonian in the Hamiltonian formulation of $\mathrm{P}_{\mathrm{V}}$ [38,

$$
\left(t h_{\mathrm{V}}^{\prime \prime}\right)^{2}-\left(h_{\mathrm{V}}-t h_{\mathrm{V}}^{\prime}+2\left(h_{\mathrm{V}}^{\prime}\right)^{2}\right)^{2}+4 \prod_{k=1}^{4}\left(h_{\mathrm{V}}^{\prime}+v_{k}\right)=0
$$

with $v_{1}+v_{2}+v_{3}+v_{4}=0$. Setting

$$
\sigma_{V}(x)+1 / 2=h_{\mathrm{V}}(t), \quad x=-\frac{i t}{2}
$$

shows that (1.6) reduces to (1.8) with $\left(v_{1}, v_{2}, v_{3}, v_{4}\right)=(1 / 2,-1 / 2,1 / 2,-1 / 2)$. Subsequently the characterisation of $\rho_{\infty}(x ; 0)$ in terms of the solution of a differential equation was extended by Its et al. 20] (see also 28) to the characterisation of $\rho_{\infty}^{T}(x ; 0)$ - the density matrix of the impenetrable Bose gas at non-zero temperature $T$, as the solution of coupled partial differential equations.

In the same study that (1.6) was obtained, Jimbo et al. evaluated the scaled probability of an eigenvalue free interval for large GUE random matrices (random 
Hermitian matrices) in terms of another particular case of the $\sigma$-form of $\mathrm{P}_{\mathrm{V}}$. In recent years there has been considerable progress in the evaluation of probabilities and averages in matrix ensembles in terms of Painlevé transcendents (see e.g. 11]). Because of the close relationship between the density matrix for impenetrable bosons and gap probabilities in matrix ensembles, the random matrix results can be used to extend the density matrix Painlevé transcendent evaluation (1.5) to the exact Painlevé transcendent of $\rho_{N}(\iota(x) ; x)$ in the four cases, where $\iota(x)$ denotes the image of $x$ reflected about the centre of the system.

We adopt two distinct strategies to obtain the exact evaluations. In Section 3 we present the first approach where we work directly with the definition of $\rho_{N}(x ; y)$ on a circle as a multidimensional integral. It turns out that this integral is one of a general class which have recently [11] been identified as $\tau$-functions for certain $\mathrm{P}_{\mathrm{VI}}$ systems. We show that our $\mathrm{P}_{\mathrm{VI}}$ transcendent evaluation for the finite system scales to the infinite system result (1.6). As well as being a special case of the class of integrals related to $\mathrm{P}_{\mathrm{VI}}$ systems in [11, the multidimensional integral formula for $\rho_{N}(x ; y)$ on a circle is also a special case of a class of integrals over the unitary group shown to satisfy integrable recurrence relations in [1]. We will show that these recurrences can alternatively be derived from orthogonal polynomial theory 33 .

Underpinning the second of our strategies is the formulation of Lenard 29] which allows $\rho_{N}(x ; y)$ to be expressed in terms of the Fredholm minor of $1-\xi K_{J}$, where $K_{J}$ is the integral operator on $J=[x, y]$ with kernel $K$ of ChristoffelDarboux type. It is this formulation which also underlies the calculation of [22] The Fredholm minor in turn can be expressed in terms of the product of the corresponding Fredholm determinant, and the resolvent kernel $R(s, t)$ evaluated at the endpoints $x, y$ of $J$. These latter two quantities have been extensively studied in the context of gap probabilities in random matrix ensembles [42,43, 49, [48, allowing us to essentially read off from the existing literature an expression for $\rho_{N}(\iota(x) ; x)$ in terms of Painlevé transcendents in each case. This is done in Section 4.

The significance of our results, from the viewpoint of the theory of the ground state occupation of single particle states for the impenetrable Bose gas, and from the viewpoint of the Painleve theory, is discussed in Section 5.

\section{Formulations of $\rho_{N}(x ; y)$}

2.1. The wave functions. We will first revise the construction of the ground state wave function for impenetrable bosons on the circle, on the line with a confining harmonic potential, and on an interval with Dirichlet or Neumann boundary conditions. The wave function and density matrix will be given a superscript "C", "H", "D" and "N" respectively to distinguish the four cases.

In general the wave function $\psi\left(x_{1}, \ldots, x_{N}\right)$ for impenetrable bosons must vanish at coincident coordinates,

$$
\psi\left(x_{1}, \ldots, x_{i}, \ldots, x_{j}, \ldots, x_{N}\right)=0 \text { for } x_{i}=x_{j},(i \neq j),
$$

and satisfy the free particle Schrödinger equation otherwise. But for point particles without spin the condition (2.1) is equivalent to the Pauli exclusion principle. This means that for any fixed ordering of the particles,

$$
x_{1}<x_{2}<\ldots<x_{N}
$$


say, there is no distinction between impenetrable bosons and free fermions [12. Consequently the ground state wave function $\psi_{0}$ can, for the ordering (2.2), be constructed out of a Slater determinant of distinct single particle states. For other orderings $\psi_{0}$ is constructed from the functional form for the sector (2.2) by the requirement that it be a symmetric function of the coordinates.

Consider the case that the particles are confined to a circle of circumference length $L$. This means we require

$$
\psi\left(x_{1}, \ldots, x_{i}+L, \ldots, x_{N}\right)=\psi\left(x_{1}, \ldots, x_{i}, \ldots, x_{N}\right)
$$

for each $i=1, \ldots, N$. Constructing a Slater determinant obeying (2.3) out of distinct single particle states with zero total momentum and minimum total energy gives

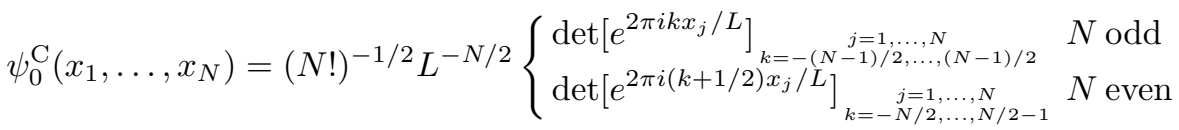

$$
\begin{aligned}
& =(N !)^{-1 / 2} L^{-N / 2} \prod_{1 \leq j<k \leq N} 2 i \sin \pi\left(x_{k}-x_{j}\right) / L
\end{aligned}
$$

where the factor of $(N !)^{-1 / 2}$ is included so that

$$
\int_{0}^{L} d x_{1} \cdots \int_{0}^{L} d x_{N}\left|\psi_{0}^{\mathrm{C}}\left(x_{1}, \ldots, x_{N}\right)\right|^{2}=1 .
$$

Excluding the (unitary) factors of $i$, and recalling (2.2), we note that this state is non-negative - a property which distinguishes the ground state in Bose systems. By the requirement that the wave function for a Bose system be symmetrical with respect to interchanges $x_{j} \leftrightarrow x_{j^{\prime}}\left(j \neq j^{\prime}\right)$ we see immediately from (2.4) that for general ordering of particles

$$
\psi_{0}^{\mathrm{C}}\left(x_{1}, \ldots, x_{N}\right)=L^{-N / 2}(N !)^{-1 / 2} \prod_{1 \leq j<k \leq N} 2\left|\sin \pi\left(x_{k}-x_{j}\right) / L\right| .
$$

In the case of impenetrable bosons on a line with a confining harmonic potential, we take as the Schrödinger operator (in reduced units)

$$
-\sum_{j=1}^{N} \frac{\partial^{2}}{\partial x_{j}^{2}}+\sum_{j=1}^{N} x_{j}^{2} .
$$

The corresponding normalised single particle eigenstates $\left\{\phi_{k}(x)\right\}_{k=0,1, \ldots}$ have the explicit form

$$
\phi_{k}(x)=\frac{2^{-k}}{c_{k}^{\mathrm{H}}} e^{-x^{2} / 2} H_{k}(x), \quad\left(c_{k}^{\mathrm{H}}\right)^{2}=\pi^{1 / 2} 2^{-k} k !
$$

where $H_{k}(x)$ denotes the Hermite polynomial of degree $k$. Forming a Slater determinant from the minimal energy states $(k=0,1, \ldots, N-1)$, making use of the Vandermonde determinant formula

$$
\operatorname{det}\left[p_{j-1}\left(x_{k}\right)\right]_{j, k=1, \ldots, N}=\operatorname{det}\left[x_{k}^{j-1}\right]_{j, k=1, \ldots, N}=\prod_{1 \leq j<k \leq N}\left(x_{k}-x_{j}\right)
$$


for any $\left\{p_{j}(x)\right\}$ with $p_{j}(x)$ a monic polynomial of degree $j$, and arguing as in going from (2.4) to (2.5) shows

$$
\psi_{0}^{\mathrm{H}}\left(x_{1}, \ldots, x_{N}\right)=\frac{1}{C_{N}^{\mathrm{H}}} \prod_{j=1}^{N} e^{-x_{j}^{2} / 2} \prod_{1 \leq j<k \leq N}\left|x_{k}-x_{j}\right|, \quad\left(C_{N}^{\mathrm{H}}\right)^{2}=N ! \prod_{l=0}^{N-1}\left(c_{l}^{\mathrm{H}}\right)^{2} .
$$

Finally we consider the case of impenetrable bosons on the interval $[0, L]$ with Dirichlet or Neumann boundary conditions, requiring that the wave function or its derivative vanishes at $x=0, L$ respectively. The single particle eigenstates $\left\{\phi_{k}(x)\right\}$ in these situations are, in increasing order of energy,

$$
\phi_{k}^{\mathrm{D}}(x)=\sqrt{\frac{2}{L}} \sin \frac{\pi k x}{L},(k=1,2, \ldots), \quad \phi_{k}^{\mathrm{N}}(x)= \begin{cases}\sqrt{\frac{1}{L}}, & k=0 \\ \sqrt{\frac{2}{L}} \cos \frac{\pi k x}{L}, & k=1, \ldots\end{cases}
$$

Recalling the $C$ and $D$ type Vandermonde formulas 40]

$$
\begin{aligned}
\operatorname{det}\left[z_{j}^{k}-z_{j}^{-k}\right]_{j, k=1, \ldots, n} & =\prod_{j=1}^{n}\left(z_{j}-z_{j}^{-1}\right) \prod_{1 \leq j<k \leq n}\left(z_{k}-z_{j}\right)\left(1-\frac{1}{z_{j} z_{k}}\right) \\
\operatorname{det}\left[z_{j}^{k-1}+z_{j}^{-(k-1)}\right]_{j, k=1, \ldots, n} & =2 \prod_{1 \leq j<k \leq n}\left(z_{k}-z_{j}\right)\left(1-\frac{1}{z_{j} z_{k}}\right)
\end{aligned}
$$

we see that the corresponding ground state wave functions are

$$
\begin{aligned}
& \psi_{0}^{\mathrm{D}}\left(x_{1}, \ldots, x_{N}\right)=\frac{1}{\sqrt{N !}}\left|\operatorname{det}\left[\phi_{k}^{\mathrm{D}}\left(x_{j}\right)\right]_{j, k=1, \ldots, N}\right| \\
& =\frac{1}{\sqrt{N !}}\left(\frac{1}{\sqrt{2 L}}\right)^{N} \prod_{l=1}^{N} 2 \sin \left(\pi x_{l} / L\right) \prod_{1 \leq j<k \leq N} 2\left|\cos \pi x_{k} / L-\cos \pi x_{j} / L\right|,
\end{aligned}
$$

and

$$
\begin{aligned}
\psi_{0}^{\mathrm{N}}\left(x_{1}, \ldots, x_{N}\right) & =\frac{1}{\sqrt{N !}}\left|\operatorname{det}\left[\phi_{k-1}^{\mathrm{N}}\left(x_{j}\right)\right]_{j, k=1, \ldots, N}\right| \\
= & \frac{1}{\sqrt{N !}} \frac{1}{\sqrt{L}}\left(\frac{1}{\sqrt{2 L}}\right)^{N-1} \prod_{1 \leq j<k \leq N} 2\left|\cos \pi x_{k} / L-\cos \pi x_{j} / L\right| .
\end{aligned}
$$

2.2. The density matrix as a determinant. The density matrix $\rho_{N+1}$ is defined as an $N$-dimensional integral by (1.1). In the cases of the impenetrable Bose gas wave functions of the previous section, this integral can be reduced to a computationally simpler $N$-dimensional determinant. For the circular case, this 
form has already been given by Lenard 29. Thus using the general Heine identity

$$
\begin{aligned}
& N ! \operatorname{det}\left[\int_{-1 / 2}^{1 / 2} d x w(z) z^{k-j}\right]_{j, k=1, \ldots, N} \\
& =\int_{-1 / 2}^{1 / 2} d x_{1} \cdots \int_{-1 / 2}^{1 / 2} d x_{N} \prod_{l=1}^{N} w\left(z_{l}\right) \prod_{1 \leq j<k \leq N}\left|z_{j}-z_{k}\right|^{2}, z_{j}:=e^{2 \pi i x_{j} / L}
\end{aligned}
$$

we see from (2.5) and (1.1) that

$$
\begin{aligned}
\rho_{N+1}^{\mathrm{C}}(x ; 0) & =\frac{1}{L} \operatorname{det}\left[a_{j-k}^{\mathrm{C}}(x)\right]_{j, k=1, \ldots, N} \\
a_{l}^{\mathrm{C}}(x) & :=\int_{-1 / 2}^{1 / 2} d t\left|e^{2 \pi i x / L}+e^{2 \pi i t}\right|\left|1+e^{2 \pi i t}\right| e^{2 \pi i l t} .
\end{aligned}
$$

Furthermore, the elements $a_{l}^{\mathrm{C}}$ have the explicit evaluation 29]

$$
\begin{aligned}
a_{0}^{\mathrm{C}} & =\frac{4}{\pi}\left[\sin \frac{\pi x}{L}+\frac{\pi}{2}\left(1-\frac{2 x}{L}\right) \cos \frac{\pi x}{L}\right] \\
a_{ \pm 1}^{\mathrm{C}} & =\frac{1}{\pi} e^{ \pm i \pi x / L}\left[\pi\left(1-\frac{2 x}{L}\right)+\sin \frac{2 \pi x}{L}\right] \\
a_{ \pm m}^{\mathrm{C}} & =\frac{4}{\pi} \frac{(-1)^{m+2}}{m\left(m^{2}-1\right)} e^{ \pm i m \pi x / L}\left[\cos \frac{\pi x}{L} \sin \frac{m \pi x}{L}-m \sin \frac{\pi x}{L} \cos \frac{m \pi x}{L}\right],|m|>1
\end{aligned}
$$

In particular, it follows that

$$
\begin{aligned}
& \rho_{1}^{\mathrm{C}}(x ; 0)=\frac{1}{L} \\
& \rho_{2}^{\mathrm{C}}(x ; 0)=\frac{4}{\pi L}\left[\pi\left(1 / 2-\frac{x}{L}\right) \cos \left(\frac{\pi x}{L}\right)+\sin \left(\frac{\pi x}{L}\right)\right] \\
& \rho_{3}^{\mathrm{C}}(x ; 0)=\frac{8}{\pi^{2} L}\left\{2-1 / 2 \pi^{2}\left(1 / 2-\frac{x}{L}\right)^{2}+3 \pi\left(1 / 2-\frac{x}{L}\right) \sin \left(\frac{\pi x}{L}\right) \cos \left(\frac{\pi x}{L}\right)\right. \\
& \left.\quad+\left[-5 / 2+2 \pi^{2}\left(1 / 2-\frac{x}{L}\right)^{2}\right] \cos ^{2}\left(\frac{\pi x}{L}\right)+1 / 2 \cos ^{4}\left(\frac{\pi x}{L}\right)\right\} .
\end{aligned}
$$

An essential ingredient underlying the applicability of (2.12) is the factorisation

$$
\psi_{0}^{\mathrm{C}}\left(x, x_{1}, \ldots, x_{N}\right)=\frac{1}{\sqrt{N+1}} \frac{1}{\sqrt{L}} \prod_{j=1}^{N}\left(2\left|\sin \left(\frac{\pi\left(x-x_{j}\right)}{L}\right)\right|\right) \psi_{0}^{\mathrm{C}}\left(x_{1}, \ldots, x_{N}\right)
$$

observed from (2.5), and their subsequent use of the determinant form in (2.4) to replace $\psi_{0}^{\mathrm{C}}$ on the right hand side. Now we observe from (2.9), (2.10), (2.11) 
that $\psi_{0}^{\mathrm{H}}, \psi_{0}^{\mathrm{D}}, \psi_{0}^{\mathrm{N}}$ in the case of $N+1$ particles can similarly be factorised. Using the general identity

$$
\begin{aligned}
& N ! \operatorname{det}\left[\int_{-\infty}^{\infty} d t\right.\left.g(t) h_{j-1}(t) h_{k-1}(t)\right]_{j, k=1, \ldots, N} \\
& \quad=\int_{-\infty}^{\infty} d x_{1} \cdots \int_{-\infty}^{\infty} d x_{N} \prod_{l=1}^{N} g\left(x_{l}\right)\left(\operatorname{det}\left[h_{j-1}\left(x_{k}\right)\right]_{j, k=1, \ldots, N}\right)^{2},
\end{aligned}
$$

(c.f. (2.12) we thus obtain analogous to (2.132.14) the determinant formulae

$$
\begin{aligned}
& \rho_{N+1}^{\mathrm{H}}(x ; y)=\frac{1}{\left(c_{N}^{\mathrm{H}}\right)^{2}} e^{-x^{2} / 2-y^{2} / 2} \operatorname{det}\left[a_{j, k}^{\mathrm{H}}(x ; y)\right]_{j, k=1, \ldots, N} \\
& \rho_{N+1}^{\mathrm{D}}(x ; y)=\frac{2}{L} \sin \frac{\pi x}{L} \sin \frac{\pi y}{L} \operatorname{det}\left[a_{j, k}^{\mathrm{D}}(x ; y)\right]_{j, k=1, \ldots, N} \\
& \rho_{N+1}^{\mathrm{N}}(x ; y)=\frac{1}{4 L} \operatorname{det}\left[a_{j, k}^{\mathrm{N}}(x ; y)\right]_{j, k=1, \ldots, N},(N \geq 1)
\end{aligned}
$$

where

$$
\begin{aligned}
& a_{j, k}^{\mathrm{H}}(x ; y)=\frac{2^{-j-k+2}}{c_{j-1}^{\mathrm{H}} c_{k-1}^{\mathrm{H}}} \int_{-\infty}^{\infty} d t|x-t||y-t| H_{j-1}(t) H_{k-1}(t) e^{-t^{2}} \\
& a_{j, k}^{\mathrm{D}}(x ; y)=8 \int_{0}^{1} d t\left|\cos \frac{\pi x}{L}-\cos \pi t\right|\left|\cos \frac{\pi y}{L}-\cos \pi t\right| \sin \pi j t \sin \pi k t \\
& a_{j, k}^{\mathrm{N}}(x ; y)=8 \int_{0}^{1} d t\left|\cos \frac{\pi x}{L}-\cos \pi t\right|\left|\cos \frac{\pi y}{L}-\cos \pi t\right| \cos \pi(j-1) t \cos \pi(k-1) t
\end{aligned}
$$

To simplify further, we note

$$
|x-t||y-t|=\left\{\begin{array}{l}
(x-t)(y-t), \quad t \notin[x, y] \\
-(x-t)(y-t), \quad t \in[x, y],
\end{array}\right.
$$

and similarly with $\left|\cos \frac{\pi x}{L}-\cos \pi t\right|\left|\cos \frac{\pi y}{L}-\cos \pi t\right|$. Use of such an identity allows $a_{j, k}^{\mathrm{H}}(x ; y)$ to be evaluated in terms of incomplete gamma functions, and $a_{j, k}^{\mathrm{D}}(x ; y), a_{j, k}^{\mathrm{N}}(x ; y)$ in a form similar to (2.15).

2.3. $\rho_{N+1}(x ; y)$ and integrals over the classical groups. In general, for a many body wave function $\psi_{0},\left|\psi_{0}\right|^{2}$ has the interpretation as a multivariable p.d.f. As first observed by Sutherland in the cases of $\psi_{0}^{\mathrm{C}}$ and $\psi_{0}^{\mathrm{H}}$, a feature of $\left|\psi_{0}\right|^{2}$ for each of the wavefunctions (2.5), (2.9), (2.10) and (2.11) is that it coincides precisely with the multivariate p.d.f. for particular classes of random matrices. Thus

$$
\begin{aligned}
& \left|\psi_{0}^{\mathrm{C}}\right|^{2}=\left.\operatorname{Ev}(U(N))\right|_{\theta=2 \pi x / L} \\
& \left|\psi_{0}^{\mathrm{H}}\right|^{2}=\operatorname{Ev}\left(\mathrm{GUE}_{N}\right) \\
& \left|\psi_{0}^{\mathrm{D}}\right|^{2}=\left.\operatorname{Ev}(S p(N))\right|_{\theta=\pi x / L} \\
& \left|\psi_{0}^{\mathrm{N}}\right|^{2}=\left.\operatorname{Ev}\left(O^{+}(2 N)\right)\right|_{\theta=\pi x / L}
\end{aligned}
$$


where $\operatorname{Ev}(X)$ denotes the eigenvalue p.d.f. of the ensemble of matrices $X$, and $U(N)$ denotes the unitary group with uniform (Haar) measure, GUE $_{N}$ the Gaussian unitary ensemble of $N \times N$ complex Hermitian matrices, $S p(N)$ the group of symplectic unitary $2 N \times 2 N$ matrices with Haar measure, and $O^{+}(2 N)$ denotes the group of real orthogonal $2 N \times 2 N$ matrices with determinant +1 and Haar measure.

Moreover, it follows from the definition (1.1) of the density matrix, and the explicit forms of the wave functions, that $\rho_{N+1}(x ; y)$ in each of the cases can be written as an average over $\operatorname{Ev}(X)$ for appropriate $X$. Explicitly

$$
\begin{aligned}
\rho_{N+1}^{\mathrm{C}}(x ; 0)= & \frac{1}{L}\left\langle\prod_{l=1}^{N}\left|2 \sin \left(\frac{\pi x}{L}-\frac{\theta_{l}}{2}\right)\right|\left|2 \sin \left(\frac{\theta_{l}}{2}\right)\right|\right\rangle_{\operatorname{Ev}(U(N))} \\
\rho_{N+1}^{\mathrm{H}}(x ; y)= & \frac{1}{\left(c_{N}^{\mathrm{H}}\right)^{2}} e^{-x^{2} / 2-y^{2} / 2}\left\langle\prod_{l=1}^{N}\left|x-x_{l}\right|\left|y-x_{l}\right|\right\rangle_{\operatorname{Ev}\left(\mathrm{GUE}_{N}\right)} \\
\rho_{N+1}^{\mathrm{D}}(x ; y)= & \frac{2}{L} \sin \frac{\pi x}{L} \sin \frac{\pi y}{L} \\
& \times\left\langle\prod_{l=1}^{N} 2\left|\cos \frac{\pi x}{L}-\cos \theta_{l}\right| 2\left|\cos \frac{\pi y}{L}-\cos \theta_{l}\right|\right\rangle_{\operatorname{Ev}(S p(N))} \\
\rho_{N+1}^{\mathrm{N}}(x ; y)= & \frac{1}{2 L}\left\langle\prod_{l=1}^{N} 2\left|\cos \frac{\pi x}{L}-\cos \theta_{l}\right| 2\left|\cos \frac{\pi y}{L}-\cos \theta_{l}\right|\right\rangle_{\operatorname{Ev}\left(O^{+}(2 N)\right)}
\end{aligned}
$$

Because it is straightforward to generate typical members from each of these matrix ensembles (see e.g. 8]), and so compute eigenvalues from the corresponding p.d.f. $\operatorname{Ev}(X)$, these expressions are well suited to evaluation via the Monte Carlo method.

For future reference we note that the density matrices for the corresponding free Fermi systems are given by the same averages, except that the absolute value signs are to be removed. In particular

$$
\rho_{N+1}^{\mathrm{C}, \mathrm{FF}}(x ; 0)=\frac{1}{L}\left\langle\prod_{l=1}^{N} 2 \sin \left(\frac{\theta_{l}}{2}-\frac{\pi x}{L}\right) 2 \sin \left(\frac{\theta_{l}}{2}\right)\right\rangle_{\operatorname{Ev}(U(N))} .
$$

Furthermore it is elementary to compute density matrices for free Fermi systems as sums over single particle states (a consequence of all energy states below the Fermi surface having occupation unity), and this implies the explicit evaluation

$$
\rho_{N+1}^{\mathrm{C}, \mathrm{FF}}(x ; 0)=\frac{1}{L} \frac{\sin (\pi(N+1) x / L)}{\sin (\pi x / L)} .
$$

2.4. Systematic small- $|x-y|$ expansion of $\rho_{N}(x ; y)$. According to the definition (1.1), the density matrix at coincident points $x=y$ is equal to the particle density. But the particle density for the impenetrable Bose gas is the same as for the corresponding free Fermi system and thus simple to compute. In the infinite system, the translational invariance of the state gives that the particle density 
is a constant. For this case Lenard [30] has shown how to make a systematic expansion of the density matrix $\rho_{\infty}(x ; y)$ about the case of coincident points $\rho_{\infty}(x ; x)$. Here we will present this expansion for finite Bose gas systems with ground state wave functions of the form

$$
\frac{1}{C} \prod_{l=1}^{N} g\left(x_{l}\right) \prod_{1 \leq j<k \leq N}\left|x_{k}-x_{j}\right| .
$$

This form includes the case of the harmonic well (2.9), and after the change of variables $\cos \pi x_{j} / L \mapsto x_{j}$ in (2.10) and (2.11) also includes the case of Dirichlet and Neumann boundary conditions.

Following Lenard [30, we note that substituting (2.32) in (1.1) and using (2.25) shows

$$
\begin{aligned}
\rho_{N}(x ; y) & =\frac{N g(x) g(y)}{C}\left(\int_{\Omega}-\xi \int_{x}^{y}\right) d x_{2} g^{2}\left(x_{2}\right) \cdots\left(\int_{\Omega} d x_{N}-\xi \int_{x}^{y}\right) d x_{N} g^{2}\left(x_{N}\right) \\
& \times\left.\prod_{l=2}^{N}\left(x-x_{l}\right)\left(y-x_{l}\right) \prod_{2 \leq j<k \leq N}\left(x_{k}-x_{j}\right)^{2}\right|_{\xi=2} .
\end{aligned}
$$

One now introduces the Fermi type distribution function

$$
\begin{aligned}
\rho_{N}^{\mathrm{FF}}\left(x ; y ; x_{2}, \ldots, x_{n}\right) & =\frac{N g(x) g(y)}{C} \prod_{l=2}^{n} g^{2}\left(x_{l}\right) \int_{\Omega} d x_{n+1} g^{2}\left(x_{n+1}\right) \cdots \int_{\Omega} d x_{N} g^{2}\left(x_{N}\right) \\
& \times \prod_{l=2}^{N}\left(x-x_{l}\right)\left(y-x_{l}\right) \prod_{2 \leq j<k \leq N}\left(x_{k}-x_{j}\right)^{2} .
\end{aligned}
$$

(when $n=1$ this corresponds to the free fermion one-body density matrix). Expanding (2.33) in a power series in $\xi$ and using the definition (2.34) shows

$$
\rho_{N}(x ; y)=\left.\sum_{n=0}^{\infty} \frac{(-\xi)^{n}}{n !} \int_{x}^{y} d x_{2} \ldots \int_{x}^{y} d x_{n+1} \rho_{N}^{\mathrm{FF}}\left(x ; y ; x_{2}, \ldots, x_{n+1}\right)\right|_{\xi=2}
$$

(the summation can be extended to infinity since $\rho^{\mathrm{FF}}\left(x ; y ; x_{2}, \ldots, x_{n}\right)=0$ for $n>N)$.

Next, let $\left\{p_{j}(x)\right\}_{j=0,1, \ldots}$ be monic polynomials of degree $j$, orthogonal with respect to the weight function $g^{2}(x)$. Then writing the integrand in (2.34) as a product of Slater determinants using (2.8) and making use of the orthogonality of the $p_{j}(x)$, a standard calculation shows

$$
\begin{aligned}
& \rho_{N}^{\mathrm{FF}}\left(x ; y ; x_{2}, \ldots, x_{n}\right)=\operatorname{det}\left[\begin{array}{cc}
K(x, y) & {\left[K\left(x_{j}, y\right)\right]_{j=2, \ldots, n}} \\
{\left[K\left(x, x_{k}\right)\right]_{k=2, \ldots, n}} & {\left[K\left(x_{j}, x_{k}\right)\right]_{j, k=2, \ldots, n}}
\end{array}\right] \\
& =: K\left(\begin{array}{llll}
x & x_{2} & \cdots & x_{n} \\
y & x_{2} & \cdots & x_{n}
\end{array}\right)
\end{aligned}
$$


where, with $\mathcal{N}_{j}:=\int_{-\infty}^{\infty} g^{2}(x)\left(p_{j}(x)\right)^{2} d x$,

$$
\begin{aligned}
& K(x, y):=g(x) g(y) \sum_{j=0}^{N-1} \frac{p_{j}(x) p_{j}(y)}{\mathcal{N}_{j}} \\
& =\frac{g(x) g(y)}{\mathcal{N}_{N-1}} \frac{p_{N}(x) p_{N-1}(y)-p_{N-1}(x) p_{N}(y)}{x-y}=\rho_{N}^{\mathrm{FF}}(x ; y) .
\end{aligned}
$$

The equality in (2.37) follows from the Christoffel-Darboux summation formula, and leads to the name Christoffel-Darboux kernel (the latter term is due to a relationship with integral equations; see Section 4.1) for (2.37). Hence

$$
\begin{aligned}
\rho_{N}(x ; y) & =\left.\sum_{n=0}^{\infty} \frac{(-\xi)^{n}}{n !} \int_{x}^{y} d x_{2} \cdots \int_{x}^{y} d x_{n+1} K\left(\begin{array}{cccc}
x & x_{2} & \cdots & x_{n+1} \\
y & x_{2} & \cdots & x_{n+1}
\end{array}\right)\right|_{\xi=2} \\
& :=-\left.\frac{1}{\xi} \Delta_{[x, y]}\left(\begin{array}{l}
x \\
y
\end{array} \xi\right)\right|_{\xi=2}
\end{aligned}
$$

where

$$
\Delta_{[x, y]}\left(\begin{array}{l}
a \\
b
\end{array} ; \xi\right):=\sum_{n=0}^{\infty} \frac{(-\xi)^{n+1}}{n !} \int_{x}^{y} d x_{2} \cdots \int_{x}^{y} d x_{n+1} K\left(\begin{array}{cccc}
a & x_{2} & \cdots & x_{n+1} \\
b & x_{2} & \cdots & x_{n+1}
\end{array}\right) .
$$

As for $|x-y|$ small each term in (2.38) is proportional to successively higher powers of $|x-y|$, this is the sought systematic small $|x-y|$ expansion of $\rho_{N}(x ; y)$. We will see in Section 4.1 that the expansion (2.38) forms the basis for Painlevé transcendent evaluations of $\rho_{N}(\iota(x), x)$ in the harmonic well, Dirichlet and Neumann boundary condition cases.

\section{Jimbo-Miwa-Okamoto $\tau$-functions and orthogonal polynomials}

In this section we will provide the finite $N$ analogue of the Jimbo, Miwa, Mori and Sato 22 Painlevé transcendent evaluation (1.5) of $\rho_{\infty}(x ; 0)$, by similarly evaluating $\rho_{N+1}^{\mathrm{C}}(x ; 0)$, and also presenting a recurrence relation in $N$ for $\rho_{N+1}^{\mathrm{C}}(x ; 0)$. Our Painlevé transcendent evaluation of $\rho_{N+1}^{\mathrm{C}}(x ; 0)$ is in terms of the solution of the Painlevé VI equation in $\sigma$-form. Let us then discuss some of the theory relating to this equation.

3.1. Hamiltonian formulation of $\mathrm{P}_{\mathrm{VI}}$ and $\tau$-function sequences. There are six Painlevé equations, labelled $\mathrm{P}_{\mathrm{I}}-\mathrm{P}_{\mathrm{VI}}$. They result (see e.g. [18]) from the project undertaken by Painlevé, Gambier and others to classify solutions to second order differential equations of the form $y^{\prime \prime}=R\left(y^{\prime}, y, t\right)$, where $R$ is rational in $y^{\prime}$, algebraic in $y$ and analytic in $t$ which are free from movable branch points. It was shown that the only such equations, excluding those which could be reduced to first order equations or to linear second order equations, are $\mathrm{P}_{\mathrm{I}}-\mathrm{P}_{\mathrm{VI}}$. Our interest is in the $\mathrm{P}_{\mathrm{VI}}$ equation, which has the form

$$
\begin{aligned}
q^{\prime \prime}=1 / 2\left(\frac{1}{q}+\right. & \left.\frac{1}{q-1}+\frac{1}{q-t}\right)\left(q^{\prime}\right)^{2}-\left(\frac{1}{t}+\frac{1}{t-1}+\frac{1}{q-t}\right) q^{\prime} \\
& +\frac{q(q-1)(q-t)}{t^{2}(t-1)^{2}}\left(\alpha+\beta \frac{t}{q^{2}}+\gamma \frac{(t-1)}{(q-1)^{2}}+\delta \frac{t(t-1)}{(q-t)^{2}}\right),
\end{aligned}
$$


and its solution, the $\mathrm{P}_{\mathrm{VI}}$ transcendent $q(t)$. We will see that $\rho_{N+1}^{\mathrm{C}}(x ; 0)$ can be identified with a $\tau$-function sequence in the $\mathrm{P}_{\mathrm{VI}}$ system.

The $\mathrm{P}_{\mathrm{VI}}$ system refers to the Hamiltonian system $\{q, p ; H, t\}$

$$
q^{\prime}=\frac{\partial H}{\partial p}, \quad p^{\prime}=-\frac{\partial H}{\partial q}
$$

where, with $\alpha_{0}+\alpha_{1}+2 \alpha_{2}+\alpha_{3}+\alpha_{4}=1$,

$$
\begin{aligned}
t(t-1) H= & q(q-1)(q-t) p^{2} \\
& -\left[\alpha_{4}(q-1)(q-t)+\alpha_{3} q(q-t)+\left(\alpha_{0}-1\right) q(q-1)\right] p \\
& +\alpha_{2}\left(\alpha_{1}+\alpha_{2}\right)(q-t) .
\end{aligned}
$$

It has been known since the work of Malmquist in the early 1920's 34 that the $\mathrm{P}_{\mathrm{VI}}$ equation (3.1) results from the Hamiltonian system (3.2), (3.3) by eliminating $p$ and choosing the parameters so that

$$
\alpha=1 / 2 \alpha_{1}^{2}, \beta=-1 / 2 \alpha_{4}^{2}, \gamma=1 /{ }_{2} \alpha_{3}^{2}, \delta=1 / 2\left(1-\alpha_{0}^{2}\right) .
$$

One sees that the Hamiltonian can be written as an explicit rational function of the $\mathrm{P}_{\mathrm{VI}}$ transcendent and its derivative. This follows from the fact that with $H$ given by (3.3), the first of the Hamilton equations is linear in $p$, so $p$ can be written as a rational function of $q, q^{\prime}$ and $t$.

The $\tau$-function is defined in terms of the Hamiltonian by

$$
H=\frac{d}{d t} \log \tau(t)
$$

The utility of being able to identify $\rho_{N+1}^{\mathrm{C}}(x ; 0)$ as a $\tau$-function for the $\mathrm{P}_{\mathrm{VI}}$ system is that $H$, and thus by integration of (3.4) $\tau(t)$, can be characterised in terms of a differential equation.

Proposition 1. [21, 37] Rewrite the parameters $\alpha_{0}, \ldots, \alpha_{4}$ of (3.3) in favour of the parameters

$$
b_{1}=1 / 2\left(\alpha_{3}+\alpha_{4}\right), b_{2}=1 / 2\left(\alpha_{4}-\alpha_{3}\right), b_{3}=1 / 2\left(\alpha_{0}+\alpha_{1}-1\right), b_{4}=1 / 2\left(\alpha_{0}-\alpha_{1}-1\right),
$$

and introduce the auxiliary Hamiltonian $h$ by

$$
\begin{aligned}
h & =t(t-1) H+e_{2}^{\prime}[\mathbf{b}] t-\frac{1}{2} e_{2}[\mathbf{b}] \\
& =t(t-1) H+\left(b_{1} b_{3}+b_{1} b_{4}+b_{3} b_{4}\right) t-\frac{1}{2} \sum_{1 \leq j<k \leq 4} b_{j} b_{k},
\end{aligned}
$$

where $e_{j}^{\prime}[\mathbf{b}]$ denotes the $j$ th degree elementary symmetric function in $b_{1}, b_{3}$ and $b_{4}$ while $e_{j}[\mathbf{b}]$ denotes the $j$ th degree elementary symmetric function in $b_{1}, \ldots, b_{4}$. The auxiliary Hamiltonian satisfies the Jimbo-Miwa-Okamoto $\sigma$-form of $\mathrm{P}_{\mathrm{VI}}$

$$
h^{\prime}\left(t(1-t) h^{\prime \prime}\right)^{2}+\left(h^{\prime}\left[2 h-(2 t-1) h^{\prime}\right]+b_{1} b_{2} b_{3} b_{4}\right)^{2}=\prod_{k=1}^{4}\left(h^{\prime}+b_{k}^{2}\right) .
$$


A self contained derivation of this result can be found in [11.

One of the main practical consequences of the Hamiltonian formulation is that it allows for a systematic construction of special solutions via Bäcklund transformations - birational mappings which leave the Hamilton equations formally unchanged [37. The elementary Bäcklund transformations form an extended affine Weyl group of type $D_{4}^{(1)}$. By composing certain of these elementary operators, shift operators can be constructed which have the effect of incrementing the $\alpha$ parameters by \pm 1 or 0 . For example, one such operator of this type, denoted $T_{3}$ in [11], has the action

$$
T_{3} \boldsymbol{\alpha}=\left(\alpha_{0}+1, \alpha_{1}+1, \alpha_{2}-1, \alpha_{3}, \alpha_{4}\right)
$$

or equivalently, after recalling (3.5)

$$
T_{3} \mathbf{b}=\left(b_{1}, b_{2}, b_{3}+1, b_{4}\right) .
$$

Although $T_{3}$ acting on $p$ and $q$ is a non-trivial rational mapping, when acting on $H, T_{3}$ has the formal action of acting only on the $\alpha$ 's,

$$
T_{3} H=\left.H\right|_{\boldsymbol{\alpha} \mapsto T_{3} \boldsymbol{\alpha}} .
$$

This motivates introducing a sequence of Hamiltonians

$$
T_{3}^{n} H=\left.H\right|_{\boldsymbol{\alpha} \mapsto T_{3}^{n} \boldsymbol{\alpha}},
$$

and a corresponding sequence of $\tau$-functions specified by

$$
T_{3}^{n} H=\frac{d}{d t} \log \tau_{3}[n], \quad \tau_{3}[n]=\tau_{3}[n](t)=\tau\left(t ; b_{1}, b_{2}, b_{3}+n, b_{4}\right) .
$$

A crucial result due to Okamoto [37, which can be derived from the specific form of the action of $T_{3}$ and $T_{3}^{-1}$ on $H, p$ and $q$ [25], is that $\tau_{3}[n]$ satisfies a particular differential recurrence relation.

Proposition 2. The $\tau$-function sequence (3.9) satisfies the Toda lattice equation

$$
\delta^{2} \log \bar{\tau}_{3}[n]=\frac{\bar{\tau}_{3}[n-1] \bar{\tau}_{3}[n+1]}{\bar{\tau}_{3}^{2}[n]}, \quad \delta=t(t-1) \frac{d}{d t}
$$

where

$$
\bar{\tau}_{3}[n]:=(t(t-1))^{\left(n+b_{1}+b_{3}\right)\left(n+b_{3}+b_{4}\right) / 2} \tau_{3}[n] .
$$

The significance of this is that an identity of Sylvester (see [35]) gives that if

$$
\bar{\tau}_{3}[0]=1,
$$

then the general solution of (3.10) is given by

$$
\bar{\tau}_{3}[n]=\operatorname{det}\left[\delta^{j+k} \bar{\tau}_{3}[1]\right]_{j, k=0,1, \ldots, n-1} .
$$

Furthermore, restricting the parameter space so that $\alpha_{2}=0$ (which corresponds to a chamber wall or reflection hyperplane in the affine $D_{4}^{(1)}$ root system), it 
has been shown by Okamoto [37 that $\bar{\tau}_{3}[1]$ is given in terms of a solution of the Gauss hypergeometric equation. Using integral solutions of the latter, the formula (3.13) was taken as the starting point by Forrester and Witte 11] in an extensive study of multidimensional integral forms of the $\tau$-function sequence $\bar{\tau}_{3}[n]$. In particular, results relating to averages of the form (2.26), equivalent to the following were established.

Proposition 3. Define

$$
\begin{aligned}
& A_{N}(u ; \omega, \mu ; \xi)= \\
& \left.\left\langle\prod_{l=1}^{N}\left(1-\xi \chi_{[0, \phi)}^{(l)}\right)\left(2 \sin \frac{\theta_{l}}{2}\right)^{2 \omega}\left(\frac{-1}{u e^{i \theta_{l}}}\right)^{\mu}\left(1-u e^{i \theta_{l}}\right)^{2 \mu}\right\rangle_{\operatorname{Ev}(U(N))}\right|_{u=e^{-i \phi}}
\end{aligned}
$$

where $z_{l}=e^{i \theta_{l}}, 0 \leq \theta_{l} \leq 2 \pi$, and

$$
\chi_{J}^{(l)}= \begin{cases}1, & \theta_{l} \in J \\ 0, & \theta_{l} \notin J .\end{cases}
$$

Let

$$
\mathbf{b}=(1 / 2(N+\omega-\mu), \omega+1 / 2(N+\omega+\mu), 1 / 2(N-\omega+\mu),-\mu-1 / 2(N+\omega+\mu)),
$$

and write

$$
C_{1}=e_{2}^{\prime}[\mathbf{b}]+\mu N, \quad C_{2}=1 / 2 e_{2}[\mathbf{b}]+\mu N
$$

(recall the definition of $e_{2}^{\prime}[\mathbf{b}]$ and $e_{2}[\mathbf{b}]$ from Proposition 1$)$. The $\mathrm{P}_{\mathrm{VI}}$ system with parameters (3.15) permits the $\tau$-function sequence

$$
\tau_{3}[N] \propto u^{N \mu / 2} A_{N}(u ; \omega, \mu ; \xi)
$$

where the proportionality factor is independent of $u$ and furthermore

$$
C_{1} u-C_{2}+u(u-1) \frac{d}{d u} \log A_{N}(u ; \omega, \mu ; \xi)=h_{\mathrm{VI}}(u ; \mathbf{b})
$$

where $h_{\mathrm{VI}}(t ; \mathbf{b})$ is an auxiliary Hamiltonian [3.6) for the $\mathrm{P}_{\mathrm{VI}}$ system with parameters (3.15). Consequently (3.17) satisfies the $\mathrm{P}_{\mathrm{VI}}$ equation in $\sigma$-form (3.7) with parameters (3.15).

To relate (3.14) to (2.26) we note that

$$
\left.\left(1-2 \chi_{[0, \phi)}^{(l)}\right)\left(\frac{-1}{u e^{i \theta_{l}}}\right)^{1 / 2}\left(1-u e^{i \theta_{l}}\right)\right|_{u=e^{-i \phi}}=2\left|\sin \frac{\left(\theta_{l}-\phi\right)}{2}\right| .
$$

Consequently

$$
\rho_{N+1}^{\mathrm{C}}(x ; 0)=\frac{1}{L} A_{N}\left(e^{2 \pi i x / L} ; 1 / 2,1 / 2 ; 2\right)
$$

where we have used the fact that $\rho_{N+1}^{\mathrm{C}}(x ; 0)$ is even in $x$. 
The choice of the parameters in (3.18) corresponding to $\rho_{N+1}^{\mathrm{C}}$ implies a special structure to the $\tau$-function sequence (3.16). First substituting (3.18) in (3.15) shows we are considering the $\mathrm{P}_{\mathrm{VI}}$ system with parameters

$$
\mathbf{b}=\left(1 /{ }_{2} N, 1+1 /{ }_{2} N, 1 / 2 N,-1-1 /{ }_{2} N\right) .
$$

As noted above, $\tau_{3}[1]$ satisfies the Gauss hypergeometric differential equation. The parameters in the latter are related to the parameters $\mathbf{b}$ by

$$
a=b_{1}+b_{4}, b=1+b_{3}+b_{4}, c=1+b_{2}+b_{4} .
$$

Substituting the special values (3.19) we see that in particular $c=1$, which is the condition for the existence of a logarithmic solution at the origin $(u=0)$. For general $N, \tau_{3}[N]$ then corresponds to a generalisation of this logarithmic solution of the Gauss hypergeometric equation. To illustrate this point, we note that with $\mathbf{b}$ given by (3.19), according to (3.16) and (3.18) we have

$$
\left.\tau_{3}[N](u) \propto u^{N / 2} \rho_{N+1}^{\mathrm{C}}(x ; 0)\right|_{u=e^{2 \pi i x / L}} .
$$

Recalling (2.17) and (2.18) we see

$$
\begin{gathered}
\tau_{3}[1](u) \propto(u+1) v+2(u-1) \\
\tau_{3}[2](u) \propto 4\left(u^{2}+u+1\right) v^{2}+12(u-1)(u+1) v \\
-u^{-1}(u-1)^{2}\left(u^{2}-14 u+1\right)
\end{gathered}
$$

where $v=\pi i-\log u$ which exhibits the further structure of being a polynomial of degree $N$ in $v$, and a Laurent polynomial in $u$ of positive degree $N$ and negative degree $N-1$.

The $\mathrm{P}_{\mathrm{VI}}$ system with parameters (3.19) also permits a $\tau$-function sequence which is strictly a polynomial. To anticipate this we relate (3.14) to the free Fermi average (2.30) by noting

$$
\left.\left(\frac{-1}{u e^{i \theta_{l}}}\right)^{1 / 2}\left(1-u e^{i \theta_{l}}\right)\right|_{u=e^{-i \phi}}=2 \sin \frac{\left(\theta_{l}-\phi\right)}{2},
$$

and so deducing

$$
\rho_{N+1}^{\mathrm{C}, \mathrm{FF}}(x ; 0)=\frac{1}{L} A_{N}\left(e^{2 \pi i x / L} ; 1 / 2,1 / 2 ; 0\right) .
$$

Recalling (3.16) and (2.31) we see that this corresponds to the $\tau$-function sequence

$$
\tau_{3}[N](u) \propto \sum_{j=0}^{N-1} u^{j} .
$$

This class of polynomial solutions is a special case of the generalised Jacobi polynomial solutions identified in [36. As a final remark on the theme of special classes of solutions to the $\mathrm{P}_{\mathrm{VI}}$ system, we note that the specification of the 
parameters (3.19) is a particular example which permits elliptic solutions [26.5], 17. More generally the latter occur when

$$
\begin{array}{ll}
t_{1}=1+b_{3}-b_{4}=2+N \in \mathbb{Z} \\
t_{2}=b_{1}+b_{2}=1+N \in \mathbb{Z} \\
t_{3}=b_{1}-b_{2}=-1 \in \mathbb{Z} \\
t_{4}=1+b_{3}+b_{4}=0 \in \mathbb{Z}
\end{array} \quad \sum_{k=1}^{4} t_{k}=2(N+1) \in 2 \mathbb{Z} .
$$

Substituting (3.18) into (3.17) of Proposition 3 and replacing $N$ by $N-1$ throughout gives the sought evaluation of $\rho_{N}^{\mathrm{C}}(x ; 0)$ in terms of a solution of the $\mathrm{P}_{\mathrm{VI}}$ equation in $\sigma$-form.

\section{Corollary 1. Define}

$$
\sigma_{N}(u):=\left.u(u-1) \frac{d}{d u} \log \rho_{N}^{\mathrm{C}}(x ; 0)\right|_{e^{2 \pi i x / L}=u}
$$

so that

$$
\rho_{N}^{\mathrm{C}}(x ; 0)=\rho_{0} \exp \left(2 \pi i \int_{0}^{x / L} \frac{d t}{e^{2 \pi i t}-1} \sigma_{N}\left(e^{2 \pi i t}\right)\right) .
$$

The quantity $\sigma_{N}(u)$ satisfies the particular $\mathrm{P}_{\mathrm{VI}} \sigma$-form differential equation

$$
\begin{aligned}
& u^{2}(u-1)^{2}\left(\sigma_{N}^{\prime \prime}\right)^{2} \\
& +\left[\sigma_{N}-(u-1) \sigma_{N}^{\prime}+1\right]\left\{4 \sigma_{N}^{\prime}\left(\sigma_{N}-u \sigma_{N}^{\prime}\right)-\left(N^{2}-1\right)\left[\sigma_{N}-(u-1) \sigma_{N}^{\prime}\right]\right\}=0
\end{aligned}
$$

subject to the boundary condition

$$
\sigma_{N}(u) \underset{u \rightarrow 1}{\sim} \frac{N^{2}-1}{12}(u-1)^{2}+\frac{\left(N^{2}-1\right)(i N-\pi)}{24 \pi}(u-1)^{3}+\ldots
$$

The formula (3.23) also holds for $\rho_{N}^{\mathrm{C}, \mathrm{FF}}(x ; 0)$, except that $\sigma_{N}^{\mathrm{FF}}(u)$ is now subject to the boundary condition

$$
\sigma_{N}^{\mathrm{FF}}(u) \underset{u \rightarrow 1}{\sim} \frac{N^{2}-1}{12}(u-1)^{2}-\frac{N^{2}-1}{24}(u-1)^{3}+\ldots
$$

Proof - This is immediate from Proposition 3 and (3.18), (3.20), except for the boundary conditions. The latter in the free Fermi case follows by substituting the exact evaluation (2.31) in (3.21). Use is also made of the free Fermi density matrix exact evaluation (2.31) to deduce the boundary condition in the impenetrable Bose gas case. Thus according to (2.36) 2.38) we have

$$
\begin{aligned}
\rho_{N}^{\mathrm{C}}(x ; 0) & =\rho_{N}^{\mathrm{CFF}}(x ; 0)-2 \int_{0}^{x} \operatorname{det}\left|\begin{array}{cc}
\rho_{N}^{\mathrm{CFF}}(x ; 0) & \rho_{N}^{\mathrm{CFF}}\left(x_{2} ; 0\right) \\
\rho_{N}^{\mathrm{CFF}}\left(x ; x_{2}\right) & \rho_{N}^{\mathrm{CFF}}\left(x_{2} ; x_{2}\right)
\end{array}\right| d x_{2}+\cdots \\
& \underset{x \rightarrow 0}{\sim} \rho_{0}\left(1-\frac{(N-1)(N+1)}{6}\left(\frac{\pi x}{L}\right)^{2}+\frac{(N-1) N(N+1)}{9 \pi}\left(\frac{\pi x}{L}\right)^{3}+\ldots\right),
\end{aligned}
$$


where the second line follows after substituting (2.31) and expanding the first term to $O\left(x^{2}\right)$ (this term only contains even powers of $x$ ), and the second term to its leading order, $O\left(x^{3}\right)$. Finally we substitute (3.25) in (3.21) to deduce the expansion (3.24).

One immediate consequence of Corollary 1 is that it allows the small $x$ expansion to easily be extended. Thus it follows that the corrections to (3.25) at order $x^{4}$ and $x^{5}$ are

$$
+\frac{(N-1)(N+1)\left[3 N^{2}-7\right]}{360}\left(\frac{\pi x}{L}\right)^{4}-\frac{(N-1) N(N+1)\left[11 N^{2}-29\right]}{1350 \pi}\left(\frac{\pi x}{L}\right)^{5}
$$

The results (1.5), (1.6) of Jimbo et al 22 follow simply from our results (3.22), (3.23). Thus defining $\sigma_{V}(t)=\lim _{N \rightarrow \infty} \sigma_{N}\left(e^{2 i t / N}\right)$ we obtain (1.5) from (3.22), while substituting $u=e^{2 i t / N}$ in (3.23), replacing $\sigma_{N}\left(e^{2 i t / N}\right)$ with $\sigma_{V}(t)$ and equating the leading order terms in $N$ (which are $O(1)$ ) to zero gives (1.6). The boundary condition (1.7) corresponds to the scaled limit of (3.24).

3.2. Orthogonal polynomials on the unit circle. A feature of a number of recent studies 10,11, 1,3,4 relating Hankel and Toeplitz determinants to Painlevé transcendents has been the characterisation of the former not only as the solution of nonlinear differential equations, but also as the solution of nonlinear difference equations. Here we will show a difference equation characterisation is also possible for $\rho_{N}^{\mathrm{C}}(x ; 0)$.

For this purpose we adopt an orthogonal polynomial approach, similar to that used in [19. The characterisation of the density matrix as a Toeplitz determinant with a non-negative and bounded symbol (2.132.14) immediately implies an underlying orthogonal polynomial system defined on the unit circle. The weight appearing in (2.14) is the special case $a=b=1 / 2$ of the generalised Jacobi weight

$$
w(z)=\frac{C}{2 \pi}|1+z|^{2 a}|1+u z|^{2 b}, \quad a, b \in \mathbb{C}, z \in \mathbb{T}
$$

where $C$ is the normalisation

$$
\frac{C}{2 \pi} \int_{\mathbb{T}} \frac{d z}{i z}|1+z|^{2 a}|1+u z|^{2 b}=1 .
$$

Associated with (3.27) is a system of orthonormal polynomials $\left\{\phi_{n}(z)\right\}_{n=0,1, \ldots}^{\infty}$,

$$
\int_{\mathbb{T}} \frac{d z}{i z} w(z) \phi_{n}(z) \overline{\phi_{m}(z)}=\delta_{m, n} .
$$

In obtaining a recurrence relation for

$$
D_{N-1}:=\operatorname{det}\left[\int_{\mathbb{T}} \frac{d z}{i z} w(z) z^{k-j}\right]_{j, k=1, \ldots, N}
$$

and thus since

$$
\rho_{N+1}^{\mathrm{C}}(x ; 0)=\left.\frac{1}{L C^{N}} D_{N-1}\right|_{a=b=1 / 2}
$$


for the density matrix, one focuses attention on the leading two coefficients $\kappa_{n}$, $l_{n}$ in $\phi_{n}(z)$, and the trailing coefficient $\phi_{n}(0)$,

$$
\phi_{n}(z)=\kappa_{n} z^{n}+l_{n} z^{n-1}+\ldots+\phi_{n}(0) .
$$

The relevance of $\kappa_{n}, \phi_{n}(0)$ are seen from the Szegö relations [41]

$$
\kappa_{n}^{2}=\frac{D_{n-1}}{D_{n}}, \quad \kappa_{n}^{2}=\sum_{k=0}^{n}\left|\phi_{k}(0)\right|^{2}
$$

which show in particular that

$$
1-\left|r_{N}\right|^{2}=\frac{D_{N-2} D_{N}}{D_{N-1}^{2}}, \quad r_{n}:=\frac{\phi_{n}(0)}{\kappa_{n}} .
$$

We will see that for the weight (3.27), the Freud equations - which are recurrence relations among the successive coefficients $\kappa_{n}, \phi_{n}(0)$ - have a special structure which leads to a recurrence equation for $r_{n}$, and thus according to (3.29) and (3.31), for $\rho_{N}^{\mathrm{C}}(x ; 0)$.

Proposition 4. Consider the special case $a=b=1 / 2$ of (3.27), in which case according to (3.29) the relation 3.31) reads

$$
1-\left|r_{N}\right|^{2}=\frac{\rho_{N+2}^{\mathrm{C}} \rho_{N}^{\mathrm{C}}}{\left(\rho_{N+1}^{\mathrm{C}}\right)^{2}}
$$

The ratios $r_{n}$, and thus via (3.32) the successive density matrices, are determined by the third order difference equation with respect to $N$

$$
\begin{aligned}
2 \cos \frac{\pi x}{L}+2 \tilde{r}_{N+1} \tilde{r}_{N}= & \frac{1-\tilde{r}_{N+1}^{2}}{\tilde{r}_{N+1}}\left[(N+3) \tilde{r}_{N+2}+(N+1) \tilde{r}_{N}\right] \\
& -\frac{1-\tilde{r}_{N}^{2}}{\tilde{r}_{N}}\left[(N+2) \tilde{r}_{N+1}+N \tilde{r}_{N-1}\right]
\end{aligned}
$$

where $r_{n}:=e^{i \pi(1-x / L) n} \tilde{r}_{n} \in \mathbb{R}$. The initial members of this sequence of $\tilde{r}_{n}$ required to start the recurrence are

$$
\begin{aligned}
& \tilde{r}_{0}=1 \\
& \tilde{r}_{1}=1 / 4 \frac{\pi-2 \pi x / L+\sin (2 \pi x / L)}{\frac{1}{2}(\pi-2 \pi x / L) \cos (\pi x / L)+\sin (\pi x / L)}
\end{aligned}
$$

(substituting these values in (3.33) with $N=0$ allows $\tilde{r}_{2}$ to be computed). Also, the initial members of the $\rho_{N}^{\mathrm{C}}$ sequence are specified by 2.16) and (2.17) (with these values given $\rho_{3}^{\mathrm{C}}$ is computed from (3.32) with $N=1$ ).

Proof - Magnus has found a recurrence relation 33 for the ratios $r_{n}$ applicable to the generalised Jacobi weight (3.27)

$$
(n+1+a+b) r_{n+1}+(n-1+a+b) \bar{u} r_{n-1}=\frac{\bar{u} \bar{l}_{n} / \kappa_{n}+l_{n} / \kappa_{n}-n(\bar{u}+1)}{1-\left|r_{n}\right|^{2}}
$$


However this involves both $r_{n}$ and $l_{n}$ and we require a further relation to determine $l_{n}$. This relation is

$$
\frac{l_{n}}{\kappa_{n}}-\bar{u} \frac{\bar{l}_{n}}{\kappa_{n}}=\frac{(a-b) n}{n+a+b}(\bar{u}-1),
$$

and follows from telescoping the identity

$$
(n+a+b+1)\left[\frac{l_{n+1}}{\kappa_{n+1}}-\bar{u} \frac{\bar{l}_{n+1}}{\kappa_{n+1}}\right]-(n+a+b)\left[\frac{l_{n}}{\kappa_{n}}-\bar{u} \frac{\bar{l}_{n}}{\kappa_{n}}\right]=(a-b)(\bar{u}-1),
$$

which in turn is derived by evaluating

$$
\int_{\mathbb{T}} \frac{d z}{i z}(1+z)(1+u z) w^{\prime}(z) \phi_{n}(z) \overline{\phi_{n}(z)}
$$

in two different ways. Then (3.33) follows after setting $a=b=1 / 2$ and extracting a phase factor of $e^{i \pi(1-x / L) n}$.

The initial conditions (3.34), (3.35) can be determined by a Gram-Schmidt type construction of the orthonormal polynomials $\left\{\phi_{n}(z)\right\}_{n=0,1, \ldots}$. First, due to the normalisation of the weight (3.28) we have $\phi_{0}(z)=1$ and thus (3.34) follows. With

$$
\langle f, g\rangle:=\left.\int_{\mathbb{T}} \frac{d z}{i z} w(z)\right|_{a=b=1 / 2} f(z) \overline{g(z)},
$$

the orthogonality $\left\langle\phi_{1}(z), \phi_{0}(z)\right\rangle=0$, explicit value $\phi_{0}(z)=1$ and (3.30) give $\kappa_{1}\langle z, 1\rangle+\phi_{1}(0)=0$. The value of $\langle z, 1\rangle$ can be read off from (2.15), thus implying (3.35).

We note that the special cases corresponding to $x$ at either the end points ( $x=0, L$ and thus $u=1)$ or the midpoint $(x=L / 2$, and thus $u=-1)$ allow simple explicit formulas for the $r_{n}$. Thus we have [19]

$$
\begin{gathered}
u=1, \quad r_{N}=(-1)^{N} \frac{1}{N+1} \\
u=-1, \quad r_{N=2 p}=\frac{1}{N+1}, \quad r_{N=2 p+1}=0
\end{gathered}
$$

which clearly satisfy (3.33). The density matrix at these points also has a closed form evaluation,

$$
\begin{aligned}
\rho_{N}^{\mathrm{C}}(0 ; 0) & =\frac{N}{L} \\
\rho_{N=2 p}^{\mathrm{C}}(L / 2 ; 0) & =\frac{1}{L}\left(\frac{4}{\pi}\right)^{N-1} 4^{(p-1)(2 p-1)} \frac{G(p+2) G^{6}(p+1) G(p)}{G^{2}(2 p+1)} \\
\rho_{N=2 p+1}^{\mathrm{C}}(L / 2 ; 0) & =\frac{1}{L}\left(\frac{4}{\pi}\right)^{N-1} 4^{p(2 p-1)} \frac{G^{4}(p+1) G^{4}(p+2)}{G^{2}(2 p+2)},
\end{aligned}
$$

where $G(x)$, the Barnes G-function, has the explicit form $G(x)=(x-2) !(x-$ $3)$ ! ..1 ! for $x \in \mathbb{Z}_{\geq 2}$. Here the former evaluation follows from the general fact that at coincident points the density matrix is equal to the particle density, while the latter makes use of results from [19] on the explicit form of the $\kappa_{n}$ 's for the 
weight (3.27), $\kappa_{2 n}=\kappa_{2 n+1}=(2 n+1) ! / 2^{2 n}(n !)^{2} \sqrt{n+1}$, in the case $u=-1$, $a=b$. The small $x$ expansion of $\rho_{N}^{\mathrm{C}}(x ; 0)$, (3.25) and (3.26), substituted into (3.32) allow the corresponding small $x$ expansion of $\tilde{r}_{n}$ to be computed up to a sign, which in turn can be determined using (3.38). This shows

$$
\tilde{r}_{n} \sim(-1)^{n}\left\{\frac{1}{n+1}+\frac{n(n+2)}{6(n+1)}\left(\frac{\pi x}{L}\right)^{2}-\frac{n(n+2)}{3 \pi}\left(\frac{\pi x}{L}\right)^{3}+\ldots\right\} .
$$

A feature of the explicit forms (3.38) is that $\left|r_{N}\right| \rightarrow 0$ as $N \rightarrow \infty$. According to (3.31) this is a necessary condition for the convergence of $\rho_{N}^{\mathrm{C}}(x ; 0)$ as $N \rightarrow \infty$. Here we note that with $\left|r_{N}\right|$ small the difference equation (3.33) simplifies to read

$$
2 \cos \frac{\pi x}{L}=B_{N+1}-B_{N}, \quad B_{N}:=\frac{(N+2) \tilde{r}_{N+1}+N \tilde{r}_{N-1}}{\tilde{r}_{N}} .
$$

It follows from (3.39) that $B_{N}=2 N \cos \pi x / L+C$, where $C$ is independent of $N$. Noting that this implies $B_{N} \sim 2(N+1) \cos \pi x / L$ for $N$ large, we thus obtain the recurrence

$$
(N+2) \tilde{r}_{N+1}+N \tilde{r}_{N-1}=(N+1) \tilde{r}_{N} 2 \cos \pi x / L,
$$

which with $A_{N}:=(N+1) \tilde{r}_{N}, \cos \pi x / L:=t$ reads

$$
A_{N+1}+A_{N-1}=2 t A_{N} \text {. }
$$

This is precisely the three term recurrence satisfied by the Chebyshev polynomials [41). Thus (3.33) can be regarded as a non-linear generalisation of the Chebyshev recurrence (3.41).

Although it is not obvious from the derivation, the equations (3.32) and (3.33) remain valid in the free Fermi case. This can be seen by substituting the exact evaluation (2.31) into (3.32) to deduce

$$
\tilde{r}_{n}=\frac{\sin \pi x / L}{\sin \pi(n+1) x / L}
$$

and then verifying that this is an exact solution of (3.33). Unlike $\tilde{r}_{n}$ in the Bose case, (3.2) does not obey the inequality $\left|\tilde{r}_{n}\right|<1$ for all $x$.

As our final point of the difference equation, we remark that recently Adler and van Moerbeke [1] have constructed essentially the same pair of coupled recurrences (3.36), (3.37) from their theory of the Toeplitz lattice and its Virasoro algebra. In the particular case at hand their weight is specialised to $\alpha=\beta=1$, and $\xi^{-2}=u=e^{2 \pi i x / L}$. Their variables are related to ours by $x_{n}=\tilde{r}_{n}$ and through the use of their relation (0.0.14), which is the analogue of (3.37), then one can show $y_{n}=x_{n}$. The other recurrence in their work, $(0.0 .15)$ is the analogue of (3.36) and can be shown to lead to

$$
\begin{aligned}
\left(1-x_{n}^{2}\right)\left[(n+2) x_{n+1} x_{n-1}+n+1\right]-\left(1-x_{n-1}^{2}\right)\left[(n-1) x_{n} x_{n-2}+n\right] \\
=1+2 x_{n-1} x_{n} \cos (\pi x / L)+x_{n-1}^{2} x_{n}^{2} \\
-1+\left(1-x_{1}^{2}\right)\left(3 x_{2}+2\right)-x_{1}\left(x_{1}+2 \cos (\pi x / L)\right) .
\end{aligned}
$$

Now by clearing denominators and rearranging (3.33) one can recover the first five terms of the above relation. Furthermore by using the initial conditions of the recurrence (3.34), (3.35) one can show that the sum of the last three terms is identically zero and thus the two forms are the same. 


\section{Painlevé-type evaluations of $\rho_{N}(\iota(x) ; x)$}

4.1. Fredholm formulation. While the unitary average (2.26) defining $\rho_{N+1}^{\mathrm{C}}(x ; 0)$ is a known $\tau$-function in the Painlevé theory, the same is not true of the averages (2.27) - (2.29). Indeed the density matrices in these cases are genuinely functions of both $x$ and $y$. These variables play the role of time in the Hamiltonian formulations of the Painlevé equations, so there being more than one time variable, we are taken outside this class. However, with $y=\iota(x)$, where $\iota(x)$ denotes the reflection of $x$ about the centre of the system (thus $\iota(x)=-x$ for the harmonic well case, and $\iota(x)=L-x$ for the case of the Dirichlet and Neumann boundary conditions) we again have a function of one variable. Although this cannot be recognised as a single $\tau$-function, it turns out that we can formulate the calculation of $\rho_{N}(\iota(x) ; x)$ so that it is expressed in terms of quantities known in terms of Painlevé transcendents from random matrix theory. For this one makes use of a classical operator theoretic interpretation of (2.38) relating to Fredholm integral equations [30].

It is the latter formulation which has been used in the pioneering work of Jimbo et al. 22 on the evaluation of the bulk density matrix in terms of a Painleve $\mathrm{V}$ transcendent, and the generalisation of this result by Its, Korepin and coworkers 20,28 to the temperature dependent bulk density matrix. The key point is that with $K_{J}$ denoting the integral operator on $J=[x, y]$ with kernel (2.37), and $R(a, b ; \xi)$ denoting the kernel of the resolvent operator $R:=$ $\xi K_{J}\left(1-\xi K_{J}\right)^{-1}$, it is true in general that (see e.g. [30]22])

$$
\Delta_{[x, y]}\left(\begin{array}{l}
a \\
b
\end{array} ;\right)=-\xi \operatorname{det}\left(1-\xi K_{J}\right) R(a, b ; \xi)
$$

(the quantity $\Delta_{[x, y]}$ is called the first Fredholm minor). Now in the harmonic well case and the cases of Dirichlet and Neumann boundary conditions (the latter two after the change of variables $\cos \pi x_{j} / L \mapsto x_{j}$ ) the wave function is of the form (2.32) with

$$
g^{2}(x)= \begin{cases}e^{-x^{2}}, & \text { harmonic well } \\ \left(1-x^{2}\right)^{ \pm 1 / 2}, & \text { Dirichlet and Neumann }\end{cases}
$$

These weights have the property of being even is $x$. This implies a special structure to (4.1) if $J$ is also chosen to be symmetrical about the origin, $J=[-x, x]$ say. Thus a consequence of $g^{2}(x)$ being even is that the orthogonal polynomials $p_{j}(x)$ are even for $j$ even and odd for $j$ odd, and this from (2.37) implies $K(a, b)=K(-a,-b)$. Using this latter property, and with $J=[-x, x]$, it is true in general that (see e.g. 42])

$$
\frac{d}{d x} \log \operatorname{det}\left(1-\xi K_{J}\right)=-2 R(x, x ; \xi)
$$

Antidifferentiating and substituting in (4.1) with $[x, y] \mapsto[-x, x]$, then substituting the result in (2.38) shows

$$
\rho_{N}(-x ; x)=\left.R(-x, x ; \xi) \exp \left(-2 \int_{0}^{x} R(t, t ; \xi) d t\right)\right|_{\xi=2} .
$$


The crucial point of the formula (4.3) is that the quantities $R(-x, x)$ and $R(t, t)$, for Christoffel-Darboux kernels corresponding to the weights (4.2) have previously been calculated in terms of Painlevé transcendents as part of studies into gap probabilities (interval $J$ free of eigenvalues) for random matrix ensembles, the GUE in the case of the harmonic well, and the JUE with $a=b= \pm 1 / 2$ in the case of Dirichlet and Neumann boundary conditions. Although (4.2) and (4.3) have general validity, the specific integrable nature of the kernel (2.37) 20] is essential for this characterisation. In the latter case the quantities in (4.3) were studied in [49, but with $J=(-1,-x] \cup[x, 1)$ rather than $J=[-x, x]$. To overcome this difference in detail, we note we can rewrite (2.33) to read

$$
\begin{gathered}
\rho_{N}(x ; y)=(1-\xi)^{N-1} \frac{N g(x) g(y)}{C}\left(\int_{\Omega}+\frac{\xi}{1-\xi}\left(\int_{-\infty}^{x}+\int_{y}^{\infty}\right)\right) d x_{2} g^{2}\left(x_{2}\right) \\
\cdots\left(\int_{\Omega}+\frac{\xi}{1-\xi}\left(\int_{-\infty}^{x}+\int_{y}^{\infty}\right)\right) d x_{N} g^{2}\left(x_{N}\right) \\
\times\left.\prod_{l=2}^{N}\left(x-x_{l}\right)\left(y-x_{l}\right) \prod_{2 \leq j<k \leq N}\left(x_{k}-x_{j}\right)^{2}\right|_{\xi=2} .
\end{gathered}
$$

Repeating the working which led to (4.3) then shows

$$
\rho_{N}(-x ; x)=\left.(-1)^{N-1} R(-x, x ; \xi) \exp \left(-2 \int_{x}^{\infty} R(t, t ; \xi) d t\right)\right|_{\xi=2}
$$

where $R$ now denotes the kernel of the resolvent operator $R=\xi K_{\bar{J}}\left(1-\xi K_{\bar{J}}\right)^{-1}$, $\bar{J}:=(-\infty,-x] \cup[x, \infty)$.

4.2. Evaluation of $\rho_{N}^{\mathrm{H}}(-x ; x)$ and $\rho_{N}^{\mathrm{D}, \mathrm{N}}(L-x ; x)$. Let us begin by specifying the quantities in (4.3) in the harmonic well case. From the above discussion, this corresponds to the interval $J$ being eigenvalue free in the GUE. For this matrix ensemble, the gap probability for both the interval $J=[-x, x]$, and the interval $J=(-\infty,-x] \cup[x, \infty)$ have been studied 43 49, allowing us to use either (4.3) or (4.4) to deduce an exact expression for $\rho_{N}^{\mathrm{H}}(-x ; x)$. For purposes of specifying the boundary condition in the corresponding differential equation, it is most convenient to use the latter.

Proposition 5. For the impenetrable Bose gas on a line, confined by a harmonic well, we have

$$
\rho_{N}^{\mathrm{H}}(-x ; x)=\left.\tilde{R}(x ; \xi) \exp \left(-2 \int_{x}^{\infty} R(t ; \xi) d t\right)\right|_{\xi=2}
$$

where with $h:=\sqrt{s^{2}-2 R^{\prime}}, R$ satisfies the equation

$$
s R^{\prime \prime}+2 R^{\prime}=2 s(s-h)-2 h \sqrt{\left(R+s R^{\prime}\right)^{2}-4 s^{2}(s-h) R-2 N s^{2}(s-h)^{2}}
$$


while $\tilde{R}$ satisfies the equation

$$
\begin{aligned}
& \left(s \tilde{R}^{\prime \prime}+2 \tilde{R}^{\prime}+8 N s \tilde{R}-24 s^{2} \tilde{R}^{2}\right)^{2} \\
& =4(s-2 \tilde{R})^{2}\left(\left(\tilde{R}+s \tilde{R}^{\prime}\right)^{2}+8 N s^{2} \tilde{R}^{2}-16 s^{3} \tilde{R}^{3}\right) .
\end{aligned}
$$

The corresponding boundary conditions are

$$
\begin{aligned}
R(s ; \xi) \underset{s \rightarrow \infty}{\sim} \tilde{R}(s ; \xi) & \underset{s \rightarrow \infty}{\sim}(\xi-1)^{N-1} \rho_{N}^{\mathrm{H}}(s ; s) \\
& \underset{s \rightarrow \infty}{\sim}(\xi-1)^{N-1} \frac{2^{N-1}}{\pi^{1 / 2}(N-1) !} s^{2 N-2} e^{-s^{2}}
\end{aligned}
$$

The two resolvent kernels are not independent being related by

$$
\frac{d}{d s} R(s ; \xi)=-2 s \tilde{R}(s ; \xi)-2 \tilde{R}^{2}(s ; \xi)
$$

so that $h=s+2 \tilde{R}(s ; \xi)$. Both resolvent kernels have been reduced to a particular $\mathrm{P}_{\mathrm{V}}$ transcendent $w(x)$ with parameters

$$
\alpha=1 /{ }_{8} N^{2}, \quad \beta=-1 / 8(N-\epsilon)^{2}, \quad \gamma=1 / 2 \epsilon, \quad \delta=-1 / 2,
$$

where $\epsilon= \pm 1$. The reductions were found to be

$$
\begin{aligned}
& R=\frac{1}{8 \sqrt{x} w(w-1)^{2}}\left\{2 x \frac{d}{d x} w+N(w-1)^{2}+(2 x-1) w+1\right\} \\
& \times\left\{2 x \frac{d}{d x} w-N(w-1)^{2}-(2 x+1) w+1\right\} \\
& \tilde{R}=-\frac{\sqrt{x}}{2 w(w-1)}\left[\epsilon \frac{d}{d x} w-w\right]+\frac{N(w-1)+\epsilon}{4 \sqrt{x} w}
\end{aligned}
$$

where $s^{2}=x$.

In the case of Dirichlet and Neumann boundary conditions we require $R(-t, t)$ and $R(t, t)$ for the symmetric JUE with $(-1,-t] \cup[t, 1)$ eigenvalue free. The differential equation satisfied by $R(t, t)$ is known from [49, but the equation for $R(-t, t)$ was not made explicit in that work. We therefore give some details of the required calculation below.

Proposition 6. The impenetrable Bose gas on the finite interval $[0, L]$ subject to Dirichlet or Neumann boundary conditions at the ends has the density matrix

$$
\rho_{N}^{\mathrm{D}, \mathrm{N}}(L-x ; x)=\left.\frac{\pi}{L} \sin \frac{\pi x}{L} R_{0}^{\mathrm{D}, \mathrm{N}}(s ; \xi) \exp \left(-2 \int_{s}^{1} d t R^{\mathrm{D}, \mathrm{N}}(t ; \xi)\right)\right|_{\substack{\xi=2 \\ s=\cos \pi x / L}}
$$

where $\sigma(s):=\left(1-s^{2}\right) R^{\mathrm{D}, \mathrm{N}}(s ; \xi)$ satisfies

$$
\begin{aligned}
& \left\{\frac{s\left(1-s^{2}\right)}{F}\left[(N+\alpha)^{2} s+2 s \sigma^{\prime}-\left(1-s^{2}\right) \sigma^{\prime \prime}\right]+\left(1+s^{2}\right) F+2(N+\alpha) s\right\}^{2} \\
& \quad-\left\{2\left(1-s^{2}\right) \sigma-2(N+\alpha) s^{2}[(N+\alpha) s+F]-s[(N+\alpha) s+F]^{2}\right\}^{2} \\
& =-4 s^{2}[(N+\alpha) s+F]^{2}\{N(N+2 \alpha)+2 s \sigma+2(N+\alpha) s[(N+\alpha) s+F]\}
\end{aligned}
$$


where $\alpha= \pm 1 / 2$ and $F:=\sqrt{(N+\alpha)^{2} s^{2}-2\left(1-s^{2}\right) \sigma^{\prime}}$ and with the boundary condition

$$
\begin{aligned}
& R^{\mathrm{D}, \mathrm{N}}(s ; \xi) \underset{s \rightarrow 1}{\sim}(\xi-1)^{N-1} \rho_{N}^{\mathrm{D}, \mathrm{N}}(s ; s) \\
& \underset{s \rightarrow 1}{\sim}(\xi-1)^{N-1} \frac{(N+\alpha) \Gamma(N+2 \alpha+1)}{2^{\alpha+1}(N-1) ! \Gamma(\alpha+1) \Gamma(\alpha+2)}(1-s)^{\alpha}
\end{aligned}
$$

and $R_{0}:=R_{0}^{\mathrm{D}, \mathrm{N}}(s ; \xi)$ satisfies

$$
\begin{aligned}
& \left\{s\left(1-s^{2}\right) R_{0}^{\prime \prime}+2\left(1-s^{2}\right)\left(1-2 s^{2}\right) R_{0}^{\prime}\right. \\
& \left.+8 s R_{0}\left(2 s R_{0}-(N+\alpha) / 2\right)\left(2 s R_{0}-N-\alpha\right)-2\left(1-s^{2}+2 \alpha^{2}\right) s R_{0}\right\}^{2} \\
& \quad=4\left\{-(N+\alpha) s+2\left(1+s^{2}\right) R_{0}\right\} \\
& \quad \times\left\{\left(1-s^{2}\right)^{2}\left(R_{0}+s R_{0}^{\prime}\right)^{2}+4\left(s R_{0}\right)^{2}\left[\left(2 s R_{0}-N-\alpha\right)^{2}-\alpha^{2}\right]\right\}
\end{aligned}
$$

with the boundary condition

$$
\begin{aligned}
& R_{0}^{\mathrm{D}, \mathrm{N}}(s ; \xi) \underset{s \rightarrow 1}{\sim}(\xi-1)^{N-1} K_{N}^{\mathrm{D}, \mathrm{N}}(-s, s) \\
& \underset{s \rightarrow 1}{\sim}(\xi-1)^{N-1} \frac{\Gamma(N+2 \alpha+1)}{2^{\alpha+1}(N-1) ! \Gamma^{2}(\alpha+1)}(1-s)^{\alpha}
\end{aligned}
$$

Proof - Both cases come under the symmetric Jacobi weight discussed in [49] however this study doesn't contain sufficient details for our purposes. From Proposition 8 of the above we can derive an integral of the system of differential equations. Combining (4.26) and (4.27) we have

$$
\left(1-s^{2}\right)(q p)^{\prime}-\left[\beta_{0}+u\left(2 \alpha_{1}-1\right)\right] p^{2}+\left[\gamma_{0}-w\left(2 \alpha_{1}+1\right)\right] q^{2}=0
$$

and we can rewrite (4.23) as

$$
\sigma-\left[\beta_{0}+u\left(2 \alpha_{1}-1\right)\right] p^{2}-\left[\gamma_{0}-w\left(2 \alpha_{1}+1\right)\right] q^{2}-2 \alpha_{1} s q p-2 s^{-1}\left(1-s^{2}\right) q^{2} p^{2}=0 .
$$

Adding (4.12) to $2 \alpha_{1}$ times (4.11) and employing $(4.24,25,28)$ we find the sum to be a perfect derivative, and given that $R_{0}, \sigma, u, w$ all vanish as $s \rightarrow 1$ the integral is

$$
\left[\beta_{0}+u\left(2 \alpha_{1}-1\right)\right]\left[\gamma_{0}-w\left(2 \alpha_{1}+1\right)\right]-2 s \sigma-4 \alpha_{1}\left(1-s^{2}\right) q p=\beta_{0} \gamma_{0}=N(N+2 \alpha) .
$$

Given this integral we can follow the procedure in the proof of Proposition 11 in [48 to derive second-order differential equations for $\sigma$ and $R_{0}$, and equations (4.5), (4.8) are the results. The boundary conditions are found from the kernels

$$
\begin{aligned}
& K_{N}(t, t)= \frac{N ! \Gamma(N+2 \alpha+1)}{2^{2 \alpha+1} \Gamma^{2}(N+\alpha)}\left(1-t^{2}\right)^{\alpha} \\
& \times\left[P_{N-1}^{(\alpha+1, \alpha)}(t) P_{N-1}^{(\alpha, \alpha+1)}(t)-P_{N}^{(\alpha, \alpha)}(t) P_{N-2}^{(\alpha+1, \alpha+1)}(t)\right] \\
& K_{N}(-t, t)=(-)^{N-1} \frac{N ! \Gamma(N+2 \alpha+1)}{2^{2 \alpha+1} \Gamma(N+\alpha) \Gamma(N+\alpha+1)} \frac{\left(1-t^{2}\right)^{\alpha}}{t} P_{N-1}^{(\alpha, \alpha)}(t) P_{N}^{(\alpha, \alpha)}(t)
\end{aligned}
$$


where the $P_{N}^{(\alpha, \beta)}(t)$ is the standard Jacobi polynomial.

Again the two resolvent kernels are connected by

$$
\frac{d}{d s} \sigma=-2(N+\alpha) s R_{0}-2\left(1-s^{2}\right) R_{0}^{2}
$$

This system was solved in terms of $\mathrm{P}_{\mathrm{VI}}$ transcendent $w(x)$ with parameters

$$
\alpha=1 / 8, \quad \beta=-1 / 2(N+\alpha-\epsilon / 2)^{2}, \quad \gamma=0, \quad \delta=1 / 2\left(1-\alpha^{2}\right)
$$

according to

$$
\begin{aligned}
R_{0}(s)= & \frac{\epsilon}{2 \sqrt{x} w} \frac{d}{d x} w-\frac{1}{4 \sqrt{x}(x-1)} \frac{w-1}{w}[\epsilon(w+x)-2(N+\alpha) x] \\
\sigma(s)=- & \frac{\left[2(x-1) \frac{d}{d x} w-(w-1)(w+x)\right]^{2}}{8 \sqrt{x} w(w-1)(w-x)} \\
& -\frac{\sqrt{x}(w-1)}{2 w(w-x)}\left[N(N+2 \alpha) w-(N+\alpha)^{2} x\right]
\end{aligned}
$$

where $s=\sqrt{x}$ and $\epsilon= \pm 1$.

We remark that the thermodynamic form of $\rho_{N}^{\mathrm{D}, \mathrm{N}}(x ; y)$ for $x$ and $y$ fixed but general has been studied in the spirit of the paper of Jimbo et al. by Kojima [27, although the final characterisations obtained (in terms of an integrable differential system) does not appear to be amenable to computation.

4.3. Thermodynamic limit. In the thermodynamic limit, Jimbo et al [22] were able to identify the first Fredholm minor directly as a $\tau$-function, and so had no need for the formula (4.3). Nonetheless it can be used to evaluate $\rho_{\infty}(x ; 0)$ in terms of a solution of (1.8), as we will now demonstrate. First, repeating the working which lead to (4.3) shows that with $K_{J}$ the integral operator on $J=(-x, x)$ with kernel $K(x, y)=\sin (x-y) / \pi(x-y)$ and $R=\xi K_{J}\left(1-\xi K_{J}\right)^{-1}$,

$$
\rho_{\infty}(-x ; x)=\left.\pi \rho_{0} R_{\infty}\left(-\pi \rho_{0} x, \pi \rho_{0} x ; \xi\right) \exp \left(-2 \int_{0}^{\pi \rho_{0} x} R_{\infty}(t, t ; \xi) d t\right)\right|_{\xi=2} .
$$

Furthermore, we know from [22] that

$$
-\frac{t}{2} R_{\infty}(1 / 4 t, 1 / 4 t ; \xi)=h_{\mathrm{V}}(-i t ;(0,0,0,0))
$$

where $h_{\mathrm{V}}(t ; \mathbf{v})$ satisfies (1.8), subject to the boundary condition

$$
h_{\mathrm{V}}(-i t ;(0,0,0,0)) \underset{t \rightarrow 0}{\sim}-\xi \frac{t}{2 \pi}-\xi^{2} \frac{t^{2}}{4 \pi^{2}},
$$

and we know too that

$$
\frac{d}{d t} R_{\infty}(t, t ; \xi)=2\left(R_{\infty}(-t, t ; \xi)\right)^{2}
$$


(see e.g. 42]). Consequently $\rho_{\infty}(x ; 0)$ can be expressed in terms of the transcendent (4.13) according to

$$
\begin{aligned}
& \rho_{\infty}(x ; 0)=\pi \rho_{0}\left(-\frac{d}{d t} \frac{h_{\mathrm{V}}(-i t ;(0,0,0,0))}{t}\right)^{1 / 2} \\
& \quad \times\left.\exp \left(\int_{0}^{t} \frac{h_{\mathrm{V}}(-i u ;(0,0,0,0))}{u} d u\right)\right|_{\substack{t=2 \pi \rho_{0} x \\
\xi=2}} .
\end{aligned}
$$

This is to be compared against the evaluation (1.5) due to Jimbo et al 22], with the substitution (1.9) and the boundary condition (1.7) generalised to be consistent with (2.38),

$$
\begin{gathered}
\rho_{\infty}(x ; 0)=\left.\rho_{0} \exp \int_{0}^{t} \frac{-1 / 2+h_{\mathrm{V}}(-i u ;(1 / 2,-1 / 2,1 / 2,-1 / 2))}{u} d u\right|_{\substack{t=2 \pi \rho_{0} x \\
\xi=2}}, \\
h_{\mathrm{V}}(-i t ;(1 / 2,-1 / 2,1 / 2,-1 / 2)) \underset{t \rightarrow 0}{\sim} \frac{1}{2}+\frac{t^{2}}{12}+\frac{i \xi t^{3}}{48 \pi} .
\end{gathered}
$$

Equating the logarithmic derivatives of (4.14) and (4.16) gives the identity

$$
\begin{aligned}
& h_{\mathrm{V}}(s ;(1 / 2,-1 / 2,1 / 2,-1 / 2)) \\
& \quad=1 / 2+h_{\mathrm{V}}(s ;(0,0,0,0))+\frac{s}{2} \frac{d}{d s} \log \left(-\frac{d}{d s} \frac{h_{\mathrm{V}}(s ;(0,0,0,0))}{s}\right) .
\end{aligned}
$$

It remains to derive this directly from the Painlevé theory. In this regard we note that an identity with similar characteristics for the $\mathrm{P}_{\mathrm{II}}$ system can be deduced from a classical result of Gambier, while the analogous result for the $\mathrm{P}_{\text {III }}$ system has only recently been found [4].

\section{Discussion}

We will conclude with a discussion of our results. In relation to $\rho_{N}^{\mathrm{C}}(x ; 0)$ the recurrence relation (3.33) allows rapid and stable tabulation for very large values of $N$ for all $x \in[0, L)$. Hence numerical evaluations of the $\lambda_{k}$ in (1.3) can be carried out. Although for fixed $k$ the leading behaviour in $N$ of $\lambda_{k}$ are known from (1.4), of interest is the convergence of the $\lambda_{k}$ (appropriately scaled) to their thermodynamic value. Furthermore, the differential equation characterisation of $\rho_{N}^{\mathrm{C}}(x ; 0)$ given in Corollary 1 is well suited to generating the power series expansion about $x=0$. This relates to the behaviour of $\lambda_{k}$ for $k$ large. A comprehensive study of such issues will be discussed in a forthcoming publication [9].

Our results show a remarkable Fermi-Bose correspondence at a mathematical level of characterising the density. It goes back to Girardeau that up to the sign under permutation of the coordinates, the ground state wave function of the 1d free Fermi and impenetrable Bose systems are identical. This means that quantities depending only on the absolute value squared of $\psi_{0}$ are the same for both systems. The density matrix is not of this type, and so distinguishes the two systems. Nonetheless, we find that the same differential and difference equations characterise $\rho_{N}^{\mathrm{C}}(x ; 0)$ for both the Bose and Fermi systems - they are 
only distinguished at this mathematical level by the boundary conditions. For $\rho_{\infty}(x ; 0)$ this same property was already observed by Jimbo et al $[22$. One would like to be able to use the differential equation to see how the different prescribed small $x$ behaviours imply different behaviours at large $x$. In the case of $\rho_{\infty}(x ; 0)$, Jimbo et al 22] were able to do this and so obtain the expansion

$$
\rho_{\infty}(x ; 0) \sim \rho_{0} \frac{A}{\sqrt{\rho_{0} x}}\left(1+\frac{1}{8\left(\pi \rho_{0} x\right)^{2}}\left[\cos \left(2 \pi \rho_{0} x\right)-1 / 4\right]+\mathrm{O}\left(\frac{1}{x^{4}}\right)\right),
$$

which was first derived by Vaidya and Tracy [44]45]. A challenging problem is to use Corollary 1 to deduce higher order terms in the expansion (1.4) of $\rho_{N}^{\mathrm{C}}(x ; 0)$ for large $N$, with $x / N$ fixed. Note that in the Fermi case this expansion can be read off from (2.31).

For the density matrix $\rho_{N}^{\mathrm{H}}(x ; y)$ (and similarly $\rho_{N}^{\mathrm{D}, \mathrm{N}}(x ; y)$ ) we have the presented determinant formulation specified by (2.19), (2.22), as well as the formulation (2.27) as an average over the eigenvalues of the GUE. Both these forms are suitable for the numerical computation of $\rho_{N}^{\mathrm{H}}(x ; y)$ for general $x$ and $y$. In the special case that $(x ; y) \mapsto(-x ; x)$ we have given an explicit functional form in terms of the transcendents related to $\mathrm{P}_{\mathrm{V}}$. This provides a much more efficient numerical scheme for the computation of $\rho_{N}^{\mathrm{H}}(-x ; x)$, and will provide a valuable test for the accuracy of Monte Carlo evaluation via (2.27).

With this achieved, the next step in the determination of the occupation numbers is the numerical solution of the integral equation (1.2). In addition to the numerical evaluation of the occupations, one would like to determine the exact leading asymptotic behaviour of $\lambda_{i}, i$ fixed, from an asymptotic expression for $\rho_{N}^{\mathrm{H}}(x ; y)$ analogous to (1.4). Thus we seek the asymptotic expansion of the determinant specified in (2.19), (2.22), which can be considered as a Hankel generalisation of a Fisher-Hartwig type Toeplitz determinant, or equivalently the asymptotic expansion of the random matrix average (2.27). On this latter viewpoint of the problem, we recall that the original Szegö theorem for the asymptotic form of the Toeplitz determinants with smooth, positive symbols has been proven by Johansson 23] starting from the analogue of the random matrix average (2.26) and then generalised to an analogous theorem for Hankel determinants relating to Jacobi averages [24]. We note too that some mappings of Fisher-Hartwig symbols in the Toeplitz case to analogous symbols in the Hankel case are known [2].

Another aspect of the present work which provides the beginning for future studies is our derivation of the recurrence relation (3.33) using orthogonal polynomial theory. As we have commented, the recurrence obtained via this method coincides with recurrence obtained by Adler and van Moerbeke using methods from soliton theory. Of course one would like to understand the underlying reason for this coincidence. Also, recent works of Borodin and Boyarchenko [3], 4, starting from a formulation in terms of the discrete Riemann-Hilbert problem, provides alternative recurrences involving discrete Painlevé equations for closely related Toeplitz determinants. It remains to understand the relationship between the different approaches.

Note added: Subsequent to the completion of this work an asymptotic form analogous to (1.4) has been derived in 9] for the harmonic well case, and from this it is deduced that, as with periodic boundary conditions, the $\lambda_{k}$ for fixed $k$ are proportional to $\sqrt{N}$. 
Acknowledgement. This research has been supported by the Australian Research Council. NSW thanks V. Korepin and A. Fetter for comments and suggestions on this work.

\section{References}

1. Adler, M. and van Moerbeke, P.: Recursion relations for unitary integrals, combinatorics and the Toeplitz lattice. math-ph/0201063

2. Basor, E. L. and Ehrhardt, T.: Some identities for determinants of structured matrices. Linear Algebra Appl. 343/344, 5-19 (2002). Special issue on structured and infinite systems of linear equations

3. Borodin, A.: Discrete gap probabilities and discrete Painlevé equations. math-ph/0111008

4. Borodin, A. and Boyarchenko, D.: Distribution of the first particle in discrete orthogonal polynomial ensembles. math-ph/0204001

5. Deift, P., Its, A., Kapaev, A. and Zhou, X.: On the algebro-geometric integration of the Schlesinger equations. Comm. Math. Phys. 203(3), 613-633 (1999)

6. Dettmer, S., Hellweg, D., Ryytty, P., Arlt, J. J., Ertmer, W., Sengstock, K., Petrov, D. S., Shlyapnikov, G. V., Kreutzmann, H., Santos, L. and Lewenstein, M.: Observation of phase fluctuations in elongated Bose-Einstein condensates. Phys. Rev. Lett. 87(16), 160406-1160406-4 (2001)

7. Fisher, M. E. and Hartwig, R. E.: Toeplitz determinants: some applications, theorems and conjectures. Ad. Chem. Phys. 15, 333-353 (1968)

8. Forrester, P. J.: Log Gases and Random Matrices. http://www.ms.unimelb.edu.au/matpjf/matpjf.html

9. Forrester, P. J., Frankel, N. E., Garoni, T. and Witte, N. S.: Finite one dimensional impenetrable Bose systems: Occupation numbers. in press Phys. Rev. A

10. Forrester, P. J. and Witte, N. S.: Application of the $\tau$-function theory of Painlevé equations to random matrices: PIV, PII and the GUE. Commun. Math. Phys. 219, 357-398 (2001)

11. Forrester, P. J. and Witte, N. S.: Application of the $\tau$-function theory of Painlevé equations to random matrices: PVI, the JUE, CyUE, cJUE and scaled limits. math-ph/0204008

12. Girardeau, M. D.: Relationship between systems of impenetrable bosons and fermions in one dimension. J. Math. Phys. 1, 516-523 (1960)

13. Girardeau, M. D., Wright, E. M. and Triscari, J. M.: Ground-state properties of a onedimensional system of hard-core bosons in a harmonic trap. Phys. Rev. A 63, 033601-1033601-6 (2001)

14. Görlitz, A., Vogels, J. M., Leanhardt, A. E., Raman, C., Gustavson, T. L., Abo-Shaeer, J. R., Chikkatur, A. P., Gupta, S., Inouye, S., Rosenband, T. and Ketterle, W.: Realization of Bose-Einstein condensates in lower dimensions. Phys. Rev. Lett. 87(13), 130402-1130402-4 (2001)

15. Greiner, M., Bloch, I., Mandel, O., Hänsch, T. W. and Esslinger, T.: Exploring phase coherence in a 2d lattice of Bose-Einstein condensates. Phys. Rev. Lett. 87(16), 1604051-60405-4 (2001)

16. Hartwig, R. E. and Fisher, M. E.: Asymptotic behavior of Toeplitz matrices and determinants. Arch. Rational Mech. Anal. 32, 190-225 (1969)

17. Hitchin, N. J.: Twistor spaces, Einstein metrics and isomonodromic deformations. J. Differential Geom. 42(1), 30-112 (1995)

18. Ince, E. L.: Ordinary Differential Equations. New York: Dover (1956)

19. Ismail, M. E. H. and Witte, N. S.: Discriminants and functional equations for polynomials orthogonal on the unit circle. J. Approx. Theory 110(2), 200-228 (2001)

20. Its, A. R., Izergin, A. G., Korepin, V. E. and Slavnov, N. A.: Differential equations for quantum correlation functions. In: Proceedings of the Conference on Yang-Baxter Equations, Conformal Invariance and Integrability in Statistical Mechanics and Field Theory, volume 4, pp. 1003-1037 (1990)

21. Jimbo, M. and Miwa, T.: Monodromy preserving deformation of linear ordinary differential equations with rational coefficients. II. Phys. D 2(3), 407-448 (1981)

22. Jimbo, M., Miwa, T., Môri, Y. and Sato, M.: Density matrix of an impenetrable Bose gas and the fifth Painlevé transcendent. Phys. D 1(1), 80-158 (1980)

23. Johansson, K.: On Szegö's asymptotic formula for Toeplitz determinants and generalizations. Bull. Sci. Math. (2) 112(3), 257-304 (1988)

24. Johansson, K.: On random matrices from the compact classical groups. Ann. of Math. (2) 145(3), 519-545 (1997) 
25. Kajiwara, K., Masuda, T., Noumi, M., Ohta, Y. and Yamada, Y.: Determinant formulas for the Toda and discrete Toda equations. Funkcialaj Ekvacioj 44, 291-307 (2001). solv-int/9908007

26. Kitaev, A. V. and Korotkin, D. A.: On solutions of the Schlesinger equations in terms of $\theta$-functions. Internat. Math. Res. Notices (17), 877-905 (1998)

27. Kojima, T.: Ground-state correlation functions for an impenetrable Bose gas with Neumann or Dirichlet boundary conditions. J. Statist. Phys. 88(3-4), 713-743 (1997)

28. Korepin, V. E., Bogoliubov, N. M. and Izergin, A. G.: Quantum inverse scattering method and correlation functions. Cambridge: Cambridge University Press (1993)

29. Lenard, A.: Momentum distribution in the ground state of the one-dimensional system of impenetrable bosons. J. Math. Phys. 5(7), 930-943 (1964)

30. Lenard, A.: One-dimensional impenetrable bosons in thermal equilibrium. J. Math. Phys. 7(7), 1268-1272 (1966)

31. Lenard, A.: Some remarks on large Toeplitz determinants. Pacific J. Math. 42, 137-145 (1972)

32. Lieb, E. H. and Liniger, W.: Exact analysis of an interacting Bose gas. I. The general solution and the ground state. Phys. Rev. (2) 130, 1605-1616 (1963)

33. Magnus, A.: MAPA3072A Special topics in approximation theory 1999-2000: Semi-classical orthogonal polynomials on the unit circle. http://www.math.ucl.ac.be/ magnus/

34. Malmquist, J.: Sur les équations différentielles du second ordre dont l'intégrale générale a ses points critiques fixes. Arkiv Mat., Astron. Fys. 18(8), 1-89 (1922)

35. Muir, T.: The Theory of Determinants in the Historical Order of Development. New York: Dover (1960)

36. Noumi, M., Okada, S., Okamoto, K. and Umemura, H.: Special polynomials associated with the Painlevé equations. II. In: Integrable systems and algebraic geometry (Kobe/Kyoto, 1997), pp. 349-372. River Edge, NJ: World Sci. Publishing (1998)

37. Okamoto, K.: Studies on the Painlevé equations. I. Sixth Painlevé equation $P_{\text {VI }}$. Ann. Mat. Pura Appl. (4) 146, 337-381 (1987)

38. Okamoto, K.: Studies on the Painlevé equations. II. Fifth Painlevé equation $P_{\mathrm{v}}$. Japan. J. Math. (N.S.) 13(1), 47-76 (1987)

39. Olshanii, M.: Atomic scattering in the presence of an external confinement and a gas of impenetrable bosons. Phys. Rev. Lett. 81(5), 938-941 (1998)

40. Proctor, R. A.: Odd symplectic groups. Invent. Math. 92(2), 307-332 (1988)

41. Szegö, G.: Orthogonal Polynomials. Colloquium Publications 23. Providence, Rhode Island: American Mathematical Society, third edition (1967)

42. Tracy, C. A. and Widom, H.: Introduction to random matrices. In: Geometric and quantum aspects of integrable systems (Scheveningen, 1992), pp. 103-130. Berlin: Springer (1993)

43. Tracy, C. A. and Widom, H.: Fredholm determinants, differential equations and matrix models. Commun. Math. Phys. 163(1), 33-72 (1994)

44. Vaidya, H. G. and Tracy, C. A.: One-particle reduced density matrix of impenetrable bosons in one dimension at zero temperature. Phys. Rev. Lett. 42(1), 3-6 (1979)

45. Vaidya, H. G. and Tracy, C. A.: One particle reduced density matrix of impenetrable bosons in one dimension at zero temperature. J. Math. Phys. 20(11), 2291-2312 (1979)

46. Widom, H.: Toeplitz determinants with singular generating functions. Amer. J. Math. 95, 333-383 (1973)

47. Witte, N. S.: New transformations for Painlevé's third transcendent. to appear Proc. Amer. Math. Soc.

48. Witte, N. S. and Forrester, P. J.: Gap probabilities in the finite and scaled Cauchy random matrix ensembles. Nonl. 13, 1965-1986 (2000)

49. Witte, N. S., Forrester, P. J. and Cosgrove, C. M.: Gap probabilities for edge intervals in finite Gaussian and Jacobi unitary matrix ensembles. Nonl. 13, 1439-1464 (2000) 